\title{
REGULARITY OF THE SZEGÖ PROJECTION ON MODEL WORM DOMAINS
}

\author{
ALESSANDRO MONGUZZI AND MARCO M. PELOSO
}

\begin{abstract}
In this paper we study the regularity of the Szegö projection on Lebesgue and Sobolev spaces on the boundary of the unbounded model worm domain $D_{\beta}^{\prime}$.

We consider the Hardy space $H^{2}\left(D_{\beta}^{\prime}\right)$. Denoting by $b D_{\beta}^{\prime}$ the boundary of $D_{\beta}^{\prime}$, it is classical that $H^{2}\left(D_{\beta}^{\prime}\right)$ can be identified with the closed subspace of $L^{2}\left(b D_{\beta}^{\prime}, d \sigma\right)$, denoted by $H^{2}\left(b D_{\beta}^{\prime}\right)$, consisting of the boundary values of functions in $H^{2}\left(D_{\beta}^{\prime}\right)$, where $d \sigma$ is the induced Lebesgue measure. The orthogonal Hilbert space projection $P: L^{2}\left(b D_{\beta}^{\prime}, d \sigma\right) \rightarrow H^{2}\left(b D_{\beta}^{\prime}\right)$ is called the Szegö projection.

Let $W^{s, p}\left(b D_{\beta}^{\prime}\right)$ denote the Lebesgue-Sobolev space on $b D_{\beta}^{\prime}$. We prove that $P$, initially defined on the dense subspace $W^{s, p}\left(b D_{\beta}^{\prime}\right) \cap L^{2}\left(b D_{\beta}^{\prime}, d \sigma\right)$, extends to a bounded operator $P$ : $W^{s, p}\left(b D_{\beta}^{\prime}\right) \rightarrow W^{s, p}\left(b D_{\beta}^{\prime}\right)$, for $1<p<\infty$ and $s \geq 0$.
\end{abstract}

Dedicated to John Ryan in occasion of his 60th birthday

\section{InTRODUCTION AND STATEMENT OF THE MAIN RESUltS}

For $\beta>\frac{\pi}{2}$ we consider the domain

$$
D_{\beta}^{\prime}=\left\{\left(z_{1}, z_{2}\right) \in \mathbb{C}^{2}:\left.\left|\operatorname{Im} z_{1}-\log \right| z_{2}\right|^{2}\left|<\frac{\pi}{2},\right| \log \left|z_{2}\right|^{2} \mid<\beta-\frac{\pi}{2}\right\} .
$$

Notice that $D_{\beta}^{\prime}$ is pseudoconvex, unbounded, and with Lipschitz boundary. This domain proved to be instrumental in establishing the irregularity of the Bergman projection [1] and the failure of hypoellipticity of the $\bar{\partial}$-Neumann problem [4] on the Diederich-Fornæss worm domain $\Omega_{\beta}$. The domain $\Omega_{\beta}$ was introduced by Diederich and Fornæss [5] as a smooth bounded pseudoconvex domain with non-trivial Nebenhülle. We refer the reader also to [11] for an in depth discussion of properties and open problems on $\Omega_{\beta}$.

Among the open problems on $\Omega_{\beta}$, we now discuss the question of the (ir)-regularity of the Szegö projection and of complex Green operator on the boundary $b \Omega_{\beta}$ of $\Omega_{\beta}$. The domain $D_{\beta}^{\prime}$ is biholomorphic to

$$
D_{\beta}=\left\{\left(w_{1}, w_{2}\right) \in \mathbb{C}^{2}: \operatorname{Re}\left(w_{1} e^{-i \log \left|w_{2}\right|^{2}}\right)>0,\left.|\log | w_{2}\right|^{2} \mid<\beta-\frac{\pi}{2}\right\},
$$

via the transformation $\left(z_{1}, z_{2}\right) \mapsto\left(e^{z_{1}}, z_{2}\right)$. D. Barrett [1] showed that $\Omega_{\beta}$ can be exhausted by dilations of $D_{\beta}$ and that a given regularity of the Bergman projection on $\Omega_{\beta}$ implies the corresponding regularity of the Bergman projection on $D_{\beta}$. He proved the asymptotic expansion of the Bergman kernel on $D_{\beta}^{\prime}$ and used the transformation rule for the Bergman kernel and

2010 Mathematics Subject Classification. 32A25, 32A36, 30H20.

Key words and phrases. Hardy spaces, Szegö kernel, Szegö projection, worm domain.

Both authors supported in part by the 2010-11 PRIN grant Real and Complex Manifolds: Geometry, Topology and Harmonic Analysis of the Italian Ministry of Education (MIUR). 
projection under biholomorphic mappings to show the irregularity of the Bergman projection on $D_{\beta}$, thus proving the irregularity of the Bergman projection on $\Omega_{\beta}$.

Our program is to study the (ir)-regularity of the Szegö projection adapting the above paradigm. Moreover, in analogy to M. Christ's work [4] and using results from [8], we would like to study the hypoellipticity of the complex Green operator associated to the worm domain by means of the (ir)-regularity of the Szegö projection. There exist several differences between the cases of Bergman and Szegö projections, most noticeably the lack of a transformation rule for the Szegö kernel and projection in complex dimension greater than 1. This paper is a step in our program. We provide a detailed study of the Hardy spaces on $D_{\beta}^{\prime}$, obtaining the expression of the Szegö kernel and proving the regularity of the Szegö projection on Lebesgue-Sobolev spaces.

We now describe our results in greater detail. For $\varepsilon>0$ we consider the subdomains

$$
D_{\beta, \varepsilon}^{\prime}=\left\{\left(z_{1}, z_{2}\right) \in \mathbb{C}^{2}:\left.\left|\operatorname{Im} z_{1}-\log \right| z_{2}\right|^{2}\left|<\frac{\pi}{2}-\varepsilon,\right| \log \left|z_{2}\right|^{2} \mid<\beta-\frac{\pi}{2}-\varepsilon\right\}
$$

and their boundaries

$$
\begin{aligned}
b D_{\beta, \varepsilon}^{\prime}= & \left\{\left(z_{1}, z_{2}\right) \in \mathbb{C}^{2}:\left.\left|\operatorname{Im} z_{1}-\log \right| z_{2}\right|^{2}\left|=\frac{\pi}{2}-\varepsilon,\right| \log \left|z_{2}\right|^{2} \mid \leq \beta-\frac{\pi}{2}-\varepsilon\right\} \\
& \cup\left\{\left(z_{1}, z_{2}\right) \in \mathbb{C}^{2}:\left.\left|\operatorname{Im} z_{1}-\log \right| z_{2}\right|^{2}\left|\leq \frac{\pi}{2}-\varepsilon,\right| \log \left|z_{2}\right|^{2} \mid=\beta-\frac{\pi}{2}-\varepsilon\right\} .
\end{aligned}
$$

For $1 \leq p<\infty$, we define the Hardy space of $D_{\beta}^{\prime}$,

$$
H^{p}=\left\{f \in \operatorname{Hol}\left(D_{\beta}^{\prime}\right): \sup _{\varepsilon>0} \int_{b D_{\beta, \varepsilon}^{\prime}}|f|^{p} d \sigma_{\varepsilon}<+\infty\right\}
$$

and set

$$
\|f\|_{H^{p}}^{p}=\sup _{\varepsilon>0} \int_{b D_{\beta, \varepsilon}^{\prime}}|f|^{p} d \sigma_{\varepsilon} .
$$

Standard basic facts of Hardy space theory give that any $f \in H^{p}$ admits a boundary value function, that we still denote by $f$, defined on $b D_{\beta}^{\prime}$ that is $p$-integrable w.r.t. $d \sigma$. Moreover, we have the equality

$$
\|f\|_{H^{p}}^{p}=\int_{b D_{\beta}^{\prime}}|f|^{p} d \sigma .
$$

Then, we can identify $H^{p}$ with a subspace of $L^{p}\left(b D_{\beta}^{\prime}, d \sigma\right)$, that is closed and that we denote by $H^{p}\left(b D_{\beta}^{\prime}\right)$. The orthogonal projection of $L^{2}\left(b D_{\beta}^{\prime}, d \sigma\right)$ onto $H^{2}\left(b D_{\beta}^{\prime}\right)$

$$
P: L^{2}\left(b D_{\beta}^{\prime}, d \sigma\right) \rightarrow H^{2}\left(b D_{\beta}^{\prime}\right)
$$

is the Szegö projection and it is described as an integral operator, whose kernel is the Szegö kernel of $D_{\beta}^{\prime}$.

We consider the Lebesgue-Sobolev spaces $W^{s, p}\left(b D_{\beta}^{\prime}\right)$ on $b D_{\beta}^{\prime}$, defined for $s \geq 0$ and $1 \leq$ $p \leq \infty$. We observe that $b D_{\beta}^{\prime}$ is a Lipschitz embedded hypersurface in $\mathbb{C}^{2}$ and therefore these spaces are defined by the standard well-known theory. More simply, $b D_{\beta}^{\prime}$ is union of 4 disjoint connected components and a set of surface measure zero, the distinguished boundary, and each of these components of positive measure is in fact a portion of a hyperplane in $\mathbb{R}^{4}$.

Our main results are the following. 
Theorem 1. The Szegö kernel of $D_{\beta}^{\prime}$ is given by

$$
K(z, w)=\sum_{j \in \mathbb{Z}} z_{2}^{j} \bar{w}_{2}^{j} k_{j}\left(z_{1}, w_{1}\right),
$$

where $z=\left(z_{1}, z_{2}\right), w=\left(w_{1}, w_{2}\right) \in D_{\beta}^{\prime}$ and

$$
\begin{gathered}
k_{j}\left(z_{1}, w_{1}\right)=\frac{1}{2 \pi} \int_{\mathbb{R}} \frac{e^{i\left(z_{1}-\bar{w}_{1}\right) \xi}}{\nu(\xi, j)} d \xi, \\
\nu(\xi, j)=\left(\beta-\frac{\pi}{2}\right) \cosh (\pi \xi) \int_{-1}^{1} e^{-\left(\beta-\frac{\pi}{2}\right)(2 \xi-(j+1)) s} \sqrt{1+4 e^{-\left(\beta-\frac{\pi}{2}\right) s}} d s \\
+2 \cosh \left[\left(\beta-\frac{\pi}{2}\right)\left(2 \xi-\left(j+\frac{1}{2}\right)\right)\right] \frac{\sinh (\pi \xi)}{\xi} .
\end{gathered}
$$

The series in (3) converges in $H^{2}\left(D_{\beta}^{\prime}\right)$ for every $\left(w_{1}, w_{2}\right)$ fixed, and uniformly in compact subsets of $D_{\beta}^{\prime} \times D_{\beta}^{\prime}$.

Theorem 2. The Szegö projection $P$, initially defined on the dense subspace $W^{s, p}\left(b D_{\beta}^{\prime}\right) \cap$ $L^{2}\left(b D_{\beta}^{\prime}, d \sigma\right)$, extends to a bounded operator

$$
P: W^{s, p}\left(b D_{\beta}^{\prime}\right) \rightarrow W^{s, p}\left(b D_{\beta}^{\prime}\right),
$$

for $1<p<\infty$ and $s \geq 0$.

Our results thus concern the Lebesgue-Sobolev regularity of the Szegö projection on certain domains in $\mathbb{C}^{2}$. Of course this is a classical and widely studied problem and there exists a vast literature. Hence, we restrict this discussion to the case of domains in $\mathbb{C}^{n}$, with $n>1$. The cases of smoothly bounded strongly pseudoconvex domains, domains of finite type in $\mathbb{C}^{2}$, and convex domains of finite type in in $\mathbb{C}^{n}$ are classical by now, and we refer to [22], 21], and [17, resp. On all such domains $\Omega$, the Szegö projection is bounded on the Lebesgue $W^{s, p}(b \Omega)$ for $1<p<\infty$ and $s \geq 0$, and it is also weak-type $(1,1)$. These results were extended by K. Koenig [10] to domains in CR manifolds on which the tangential Cauchy-Riemann operator has closed range and satisfying some conditions on the eigenvalues of the Levi form.

More recently, L. Lanzani and E. M. Stein studied the regularity of the Szegö projection on strongly pseudoconvex domains with minimal boundary regularity, [15, 16], obtaining the $L^{p}$-boundedness for $1<p<\infty$.

The domain $D_{\beta}^{\prime}$ is unbounded and its boundary consists of hyperplanes, hence it is Levi flat. On such domain, S. Krantz and the second author proved that the Bergman projection is bounded on $L^{p}$ for $1<p<\infty$ [12] and the first author extended to the scale of Sobolev spaces [18. As mentioned above, $D_{\beta}^{\prime}$ is biholomorphically equivalent to another model worm domain $D_{\beta}$. On $D_{\beta}$, in contrast, we expect the Szegö projection to show some irregularity, as in the case of the Bergman projection. In principle this should indicate that the Szegö projection on the smooth worm domain $\Omega_{\beta}$ would not preserve Sobolev spaces $W^{s}$ for $s \geq s_{0}$ where $s_{0}$ is a positive real number depending on the geometry of $\Omega_{\beta}$, namely, $s_{0} \rightarrow 0$ as $\beta \rightarrow+\infty$. These investigations are part of our program and are going to be topics of future work.

We mention that on the domains $D_{\beta}^{\prime}$ and $D_{\beta}$ it is possible to introduce and study also Hardy spaces defined in terms of the induced surface measure on the distinguished boundary, that is, 
in the case of the domain $D_{\beta}^{\prime}$, the four vertices in Figure 1. This has been done by the first author [19] in the case of $D_{\beta}^{\prime}$, and in [20] in the case of $D_{\beta}$. More precisely, denote by $\partial D_{\beta}^{\prime}$ and $\partial D_{\beta}$ the distinguished boundaries of $D_{\beta}^{\prime}$ and $D_{\beta}$, resp., and by $\mathscr{P}^{\prime}$ and $\mathscr{P}$ the corresponding projections, resp. We point out that in this setting, the operators $\mathscr{P}^{\prime}$ and $\mathscr{P}$ are given by singular integrals over $\partial D_{\beta}^{\prime}$ and $\partial D_{\beta}$, resp. Then, the following results are proved: $\mathscr{P}^{\prime}: W^{s, p}\left(\partial D_{\beta}^{\prime}\right) \rightarrow$ $W^{s, p}\left(\partial D_{\beta}^{\prime}\right)$, when $1<p<\infty$ and $s \geq 0([19])$, while $\mathscr{P}: L^{p}\left(\partial D_{\beta}\right) \rightarrow L^{p}\left(\partial D_{\beta}\right)$, if and only if $p \in\left(\frac{2}{1+\nu_{\beta}}, \frac{2}{1-\nu_{\beta}}\right)$, where $\nu_{\beta}=\pi /(2 \beta-\pi)([20])$.

It would also be interesting studying the regularity of the Szegö projection on domains that are models for the higher-dimensional worm domains introduced by Barrett and Şahutoğlu in [3].

For other recent papers related to the topic of this work we also mention [14, 2, 13].

The plan of the paper is the following. In Section 2 we obtain a representation of the Szegö kernel of $D_{\beta}^{\prime}$ and prove Theorem 1. In Section 3 we decompose the Szegö projection as sum of operators $T_{\ell, \ell^{\prime}}, \ell, \ell^{\prime} \in\{1, \ldots, 4\}$, and prove their boundedness on $L^{p}$ spaces. We find particularly interesting that the operators $T_{\ell, \ell^{\prime}}$ can be written as composition of Fourier multiplier operators on $\mathbb{R} \times \mathbb{T}$ and an integral operator of Hilbert type. We can then apply a combination of the classical Marcinkiewicz multiplier theorem and Schur's test to obtain the $L^{p}$-boundedness of $P$. In Section 4 we complete the proof of Theorem 2 and prove a few remaining technical lemmas.

Acknowledgments. We laid the groundwork of this project when we were both visiting the Department of Mathematical Sciences of University of Arkansas. We wish to thank such institution for the great hospitality and for providing a very stimulating working environment.

The second author wishes to thank in particular John Ryan for the many interesting and pleasant meetings and discussions had during his stay at the Department of Mathematical Sciences.

We also thanks the anonymous reviewers for carefully reading the manuscripts and making a number of remarks that helped to improve the clarity of the paper.

\section{The Hardy SPACE And the Szegö Kernel}

2.1. Decomposition of the Hardy space. In order to describe the norm on the Hardy spaces, we begin by computing $d \sigma_{\varepsilon}$. We label the sides of $b D_{\beta, \varepsilon}^{\prime}$ as $E_{1, \varepsilon}, \ldots, E_{4, \varepsilon}$, where $E_{1, \varepsilon}$ is the right side, and then proceeding counter-clockwise. Explicitly:

$$
\begin{aligned}
& E_{1, \varepsilon}=\left\{\left(z_{1}, z_{2}\right) \in \mathbb{C}^{2}: \operatorname{Im} z_{1}-\log \left|z_{2}\right|^{2}=\frac{\pi}{2}-\varepsilon,\left.|\log | z_{2}\right|^{2} \mid \leq \beta-\frac{\pi}{2}-\varepsilon\right\} ; \\
& E_{2, \varepsilon}=\left\{\left(z_{1}, z_{2}\right) \in \mathbb{C}^{2}:\left.\left.\left|\operatorname{Im} z_{1}-\log \right| z_{2}\right|^{2}\left|\leq \frac{\pi}{2}-\varepsilon, \log \right| z_{2}\right|^{2}=\beta-\frac{\pi}{2}-\varepsilon\right\} ; \\
& E_{3, \varepsilon}=\left\{\left(z_{1}, z_{2}\right) \in \mathbb{C}^{2}: \operatorname{Im} z_{1}-\log \left|z_{2}\right|^{2}=-\frac{\pi}{2}+\varepsilon,\left.|\log | z_{2}\right|^{2} \mid \leq \beta-\frac{\pi}{2}-\varepsilon\right\} ; \\
& E_{4, \varepsilon}=\left\{\left(z_{1}, z_{2}\right) \in \mathbb{C}^{2}:\left.\left.\left|\operatorname{Im} z_{1}-\log \right| z_{2}\right|^{2}\left|\leq \frac{\pi}{2}-\varepsilon, \log \right| z_{2}\right|^{2}=-\left(\beta-\frac{\pi}{2}-\varepsilon\right)\right\} .
\end{aligned}
$$

We also use the analogous decomposition on $b D_{\beta}^{\prime}$ and denote the four sides by $E_{1}, \ldots, E_{4}$. See Figure 1.

We wish to determine the induced surface measure on $b D_{\beta, \varepsilon}^{\prime}$, for $\varepsilon \geq 0$. It is well known that, if $M$ is an embedded manifold in $\mathbb{R}^{n}$, parametrized by $F: A \subset \mathbb{R}^{m} \rightarrow \mathbb{R}^{n}$, then

$$
\int_{M} f d \sigma=\int_{A} f(F(s)) \sqrt{\operatorname{det}\left({ }^{t}(J F) J F\right)(s)} d s,
$$


where $J F$ denotes the jacobian matrix of $F$.

In the case of $E_{1, \varepsilon}$ and $E_{3, \varepsilon}$, resp., we have

$$
F(x, r, \theta)=\left(\begin{array}{c}
x \\
\log r^{2}+\frac{\pi}{2}-\varepsilon \\
r \cos \theta \\
r \sin \theta
\end{array}\right), \quad \text { and } F(x, r, \theta)=\left(\begin{array}{c}
x \\
\log r^{2}-\frac{\pi}{2}+\varepsilon \\
r \cos \theta \\
r \sin \theta
\end{array}\right) \text {, resp. }
$$

with domain $A=\mathbb{R} \times\left(e^{-\frac{1}{2}\left(\beta-\frac{\pi}{2}-\varepsilon\right)}, e^{\frac{1}{2}\left(\beta-\frac{\pi}{2}-\varepsilon\right)}\right) \times(0,2 \pi)$, so that $d \sigma=d x r \sqrt{1+4 r^{-2}} d r d \theta$ (in both cases).

Analogously, in the case of $E_{2, \varepsilon}$ and $E_{4, \varepsilon}$, resp., we have

$$
F(x, y, \theta)=\left(\begin{array}{c}
x \\
y \\
e^{\frac{1}{2}\left(\beta-\frac{\pi}{2}-\varepsilon\right)} \cos \theta \\
e^{\frac{1}{2}\left(\beta-\frac{\pi}{2}-\varepsilon\right)} \sin \theta
\end{array}\right) \quad \text { and } F(x, y, \theta)=\left(\begin{array}{c}
x \\
y \\
e^{-\frac{1}{2}\left(\beta-\frac{\pi}{2}-\varepsilon\right)} \cos \theta \\
e^{-\frac{1}{2}\left(\beta-\frac{\pi}{2}-\varepsilon\right)} \sin \theta
\end{array}\right) \text {,resp. }
$$

with domain $A=\mathbb{R} \times\left\{\left|y-\left(\beta-\frac{\pi}{2}-\varepsilon\right)\right|<\frac{\pi}{2}\right\} \times(0,2 \pi)$, and $A=\mathbb{R} \times\left\{\left|y+\left(\beta-\frac{\pi}{2}-\varepsilon\right)\right|<\frac{\pi}{2}\right\} \times(0,2 \pi)$, resp. Therefore, $d \sigma=d x d y e^{\frac{1}{2}\left(\beta-\frac{\pi}{2}-\varepsilon\right)} d \theta$ and $d \sigma=d x d y e^{-\frac{1}{2}\left(\beta-\frac{\pi}{2}-\varepsilon\right)} d \theta$, resp.

We observe that the four vertices of the parallelogram in Figure 1 have zero surface measure and therefore can be disregarded and we shall do so in what follows.

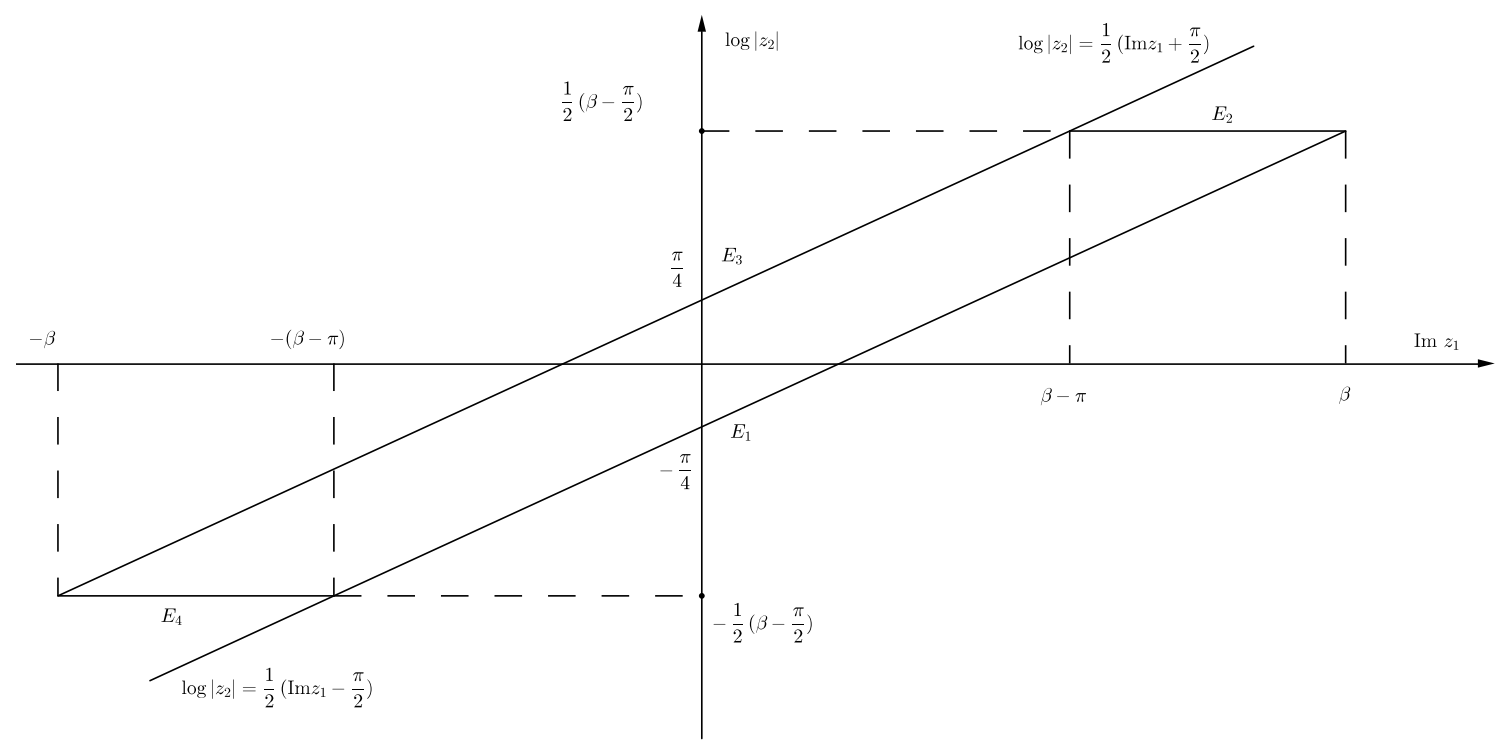

Figure 1. A representation of the domain $D_{\beta}^{\prime}$ in the $\left(\operatorname{Im} z_{1}, \log \left|z_{2}\right|\right)$-plane. 
Therefore, $f \in H^{p}$ if and only if $\|f\|_{H^{p}}^{p}=\sup _{\varepsilon>0} \int_{b D_{\beta, \varepsilon}^{\prime}}|f|^{p} d \sigma_{\varepsilon}<\infty$, where

$$
\begin{aligned}
\int_{b D_{\beta, \varepsilon}^{\prime}}|f|^{p} d \sigma_{\varepsilon}= & \left(\int_{E_{1, \varepsilon}}+\int_{E_{2, \varepsilon}}+\int_{E_{3, \varepsilon}}+\int_{E_{4, \varepsilon}}\right)|f|^{p} d \sigma_{\varepsilon} \\
= & \int_{0}^{2 \pi} \int_{e^{-\frac{1}{2}\left(\beta-\frac{\pi}{2}-\varepsilon\right)}}^{e^{\frac{1}{2}\left(\beta-\frac{\pi}{2}-\varepsilon\right)}} \int_{\mathbb{R}}\left|f\left(x+i\left(\log r^{2}+\frac{\pi}{2}-\varepsilon\right), r e^{i \theta}\right)\right|^{p} d x \sqrt{1+4 r^{-2}} r d r d \theta \\
& +\int_{0}^{2 \pi} \int_{\mathbb{R}} \int_{\left|y-\left(\beta-\frac{\pi}{2}-\varepsilon\right)\right|<\frac{\pi}{2}}\left|f\left(x+i y, e^{\frac{1}{2}\left(\beta-\frac{\pi}{2}-\varepsilon\right)} e^{i \theta}\right)\right|^{p} d y d x e^{\frac{1}{2}\left(\beta-\frac{\pi}{2}-\varepsilon\right)} d \theta \\
& +\int_{0}^{2 \pi} \int_{e^{-\frac{1}{2}\left(\beta-\frac{\pi}{2}-\varepsilon\right)}}^{e^{\frac{1}{2}\left(\beta-\frac{\pi}{2}-\varepsilon\right)}} \int_{\mathbb{R}}\left|f\left(x+i\left(\log r^{2}-\frac{\pi}{2}+\varepsilon\right), r e^{i \theta}\right)\right|^{p} d x \sqrt{1+4 r^{-2}} r d r d \theta \\
& +\int_{0}^{2 \pi} \int_{\mathbb{R}} \int_{\left|y+\left(\beta-\frac{\pi}{2}-\varepsilon\right)\right|<\frac{\pi}{2}}\left|f\left(x+i y, e^{-\frac{1}{2}\left(\beta-\frac{\pi}{2}-\varepsilon\right)} e^{i \theta}\right)\right|^{p} d y d x e^{-\frac{1}{2}\left(\beta-\frac{\pi}{2}-\varepsilon\right)} d \theta .
\end{aligned}
$$

Using standard one-variable results, it is easy to see that the above sup is attained as $\varepsilon \rightarrow 0^{+}$. Denoting by $f$ also the boundary value function of $f$, we then have

$$
\begin{aligned}
\|f\|_{H^{p}}^{p}= & \int_{0}^{2 \pi} \int_{e^{-\frac{1}{2}\left(\beta-\frac{\pi}{2}\right)}}^{e^{\frac{1}{2}\left(\beta-\frac{\pi}{2}\right)}} \int_{\mathbb{R}}\left|f\left(x+i\left(\log r^{2}+\frac{\pi}{2}\right), r e^{i \theta}\right)\right|^{p} d x r \sqrt{1+4 r^{-2}} d r d \theta \\
+ & e^{\frac{1}{2}\left(\beta-\frac{\pi}{2}\right)} \int_{0}^{2 \pi} \int_{\mathbb{R}} \int_{\left|y-\left(\beta-\frac{\pi}{2}\right)\right|<\frac{\pi}{2}}\left|f\left(x+i y, e^{\frac{1}{2}\left(\beta-\frac{\pi}{2}\right)} e^{i \theta}\right)\right|^{p} d y d x d \theta \\
& +\int_{0}^{2 \pi} \int_{e^{-\frac{1}{2}\left(\beta-\frac{\pi}{2}\right)}}^{e^{\frac{1}{2}\left(\beta-\frac{\pi}{2}\right)}} \int_{\mathbb{R}}\left|f\left(x+i\left(\log r^{2}-\frac{\pi}{2}\right), r e^{i \theta}\right)\right|^{p} d x r \sqrt{1+4 r^{-2}} d r d \theta \\
& +e^{-\frac{1}{2}\left(\beta-\frac{\pi}{2}\right)} \int_{0}^{2 \pi} \int_{\mathbb{R}} \int_{\left|y+\left(\beta-\frac{\pi}{2}\right)\right|<\frac{\pi}{2}}\left|f\left(x+i y, e^{-\frac{1}{2}\left(\beta-\frac{\pi}{2}\right)} e^{i \theta}\right)\right|^{p} d y d x d \theta \\
+ & \int_{0}^{2 \pi} \int_{-\beta+\pi}^{\beta} \int_{\mathbb{R}}\left|f\left(x+i y, e^{\frac{1}{2}\left(y-\frac{\pi}{2}\right)} e^{i \theta}\right)\right|^{p} d x e^{y-\frac{\pi}{2}} \sqrt{1+4 e^{-\left(y-\frac{\pi}{2}\right)}} d y d \theta \\
+ & e^{\frac{1}{2}\left(\beta-\frac{\pi}{2}\right)} \int_{0}^{2 \pi} \int_{\beta-\pi}^{\beta} \int_{\mathbb{R}}\left|f\left(x+i y, e^{\frac{1}{2}\left(\beta-\frac{\pi}{2}\right)} e^{i \theta}\right)\right|^{p} d x d y d \theta \\
& +\frac{1}{2} \int_{0}^{2 \pi} \int_{-\beta}^{\beta-\pi} \int_{\mathbb{R}}\left|f\left(x+i y, e^{\frac{1}{2}\left(y+\frac{\pi}{2}\right)} e^{i \theta}\right)\right|^{p} d x e^{y+\frac{\pi}{2}} \sqrt{1+4 e^{-\left(y+\frac{\pi}{2}\right)}} d y d \theta \\
& +e^{-\frac{1}{2}\left(\beta-\frac{\pi}{2}\right)} \int_{0}^{2 \pi} \int_{-\beta}^{-\beta+\pi} \int_{\mathbb{R}}\left|f\left(x+i y, e^{-\frac{1}{2}\left(\beta-\frac{\pi}{2}\right)} e^{i \theta}\right)\right|^{p} d x d y d \theta .
\end{aligned}
$$

We consider the Hilbert space $H^{2}$ and decompose it following [9], see also [1, 12]. Using Fourier series expansion in the second variable we have the orthogonal decomposition

$$
H^{2}=\bigoplus_{j \in \mathbb{Z}} \mathscr{H}_{j}
$$


where for $j \in \mathbb{Z}, \mathscr{H}_{j}$ is the subspace of functions $f$ in $H^{2}$ such that $f\left(z_{1}, e^{i \theta} z_{2}\right)=e^{i j \theta} f\left(z_{1}, z_{2}\right)$. The orthogonal projection $Q_{j}: H^{2} \rightarrow \mathscr{H}_{j}$ is

$$
Q_{j} f\left(z_{1}, z_{2}\right)=\frac{1}{2 \pi} \int_{0}^{2 \pi} f\left(z_{1}, z_{2} e^{i \theta}\right) e^{-i j \theta} d \theta
$$

The function

$$
\left(\frac{1}{2 \pi} \int_{0}^{2 \pi} f\left(z_{1}, z_{2} e^{i \theta}\right) e^{-i j \theta} d \theta\right) z_{2}^{-j}
$$

is holomorphic in $D_{\beta}^{\prime}$ and locally constant in $\left|z_{2}\right|$. Since the fibers over $z_{1}$ are connected, it is actually constant in $z_{2}$. Hence, we simply write $f_{j}\left(z_{1}\right)$, and notice that $f_{j} \in \operatorname{Hol}\left(S_{\beta}\right)$, where

$$
S_{\beta}=\{x+i y \in \mathbb{C}:|y|<\beta\} .
$$

Thus,

$$
\mathscr{H}_{j}=\left\{f \in H^{2}: f\left(z_{1}, z_{2}\right)=f_{j}\left(z_{1}\right) z_{2}^{j}, \quad \text { with } f_{j} \in \operatorname{Hol}\left(S_{\beta}\right)\right\} .
$$

Now, let $f\left(z_{1}, z_{2}\right)=\sum_{j \in \mathbb{Z}} f_{j}\left(z_{1}\right) z_{2}^{j}$. By orthogonality we have $\|f\|_{H^{2}}^{2}=\sum_{j \in \mathbb{Z}}\left\|f_{j} z_{2}^{j}\right\|_{H^{2}}^{2}$. From (41) we compute

$$
\begin{aligned}
\left\|f_{j} z_{2}^{j}\right\|_{H^{2}}^{2}= & \pi \int_{-\beta+\pi}^{\beta} \int_{\mathbb{R}}\left|f_{j}(x+i y)\right|^{2} d x e^{\left(y-\frac{\pi}{2}\right)(j+1)} \sqrt{1+4 e^{-\left(y-\frac{\pi}{2}\right)}} d y \\
+ & 2 \pi e^{\left(\beta-\frac{\pi}{2}\right)\left(j+\frac{1}{2}\right)} \int_{\mathbb{R}} \int_{\beta-\pi}^{\beta}\left|f_{j}(x+i y)\right|^{2} d y d x \\
& +\pi \int_{-\beta}^{\beta-\pi} \int_{\mathbb{R}}\left|f_{j}(x+i y)\right|^{2} d x e^{\left(y+\frac{\pi}{2}\right)(j+1)} \sqrt{1+4 e^{-\left(y+\frac{\pi}{2}\right)}} d y \\
& \quad+2 \pi e^{-\left(\beta-\frac{\pi}{2}\right)\left(j+\frac{1}{2}\right)} \int_{\mathbb{R}} \int_{-\beta}^{-\beta+\pi}\left|f_{j}(x+i y)\right|^{2} d y d x \\
= & \int_{S_{\beta}}\left|f_{j}\left(z_{1}\right)\right|^{2} \omega_{j}(y) d A\left(z_{1}\right)
\end{aligned}
$$

where $z_{1}=x+i y$, and

$$
\begin{aligned}
\omega_{j}(y)=\pi \chi_{(-\beta+\pi, \beta)}(y) e^{\left(y-\frac{\pi}{2}\right)(j+1)} \sqrt{1+4 e^{-\left(y-\frac{\pi}{2}\right)}}+2 \pi \chi_{(\beta-\pi, \beta)}(y) e^{\left(\beta-\frac{\pi}{2}\right)\left(j+\frac{1}{2}\right)} \\
\quad+\pi \chi_{(-\beta, \beta-\pi)}(y) e^{\left(y+\frac{\pi}{2}\right)(j+1)} \sqrt{1+4 e^{-\left(y+\frac{\pi}{2}\right)}}+2 \pi \chi_{(-\beta,-\beta+\pi)}(y) e^{-\left(\beta-\frac{\pi}{2}\right)\left(j+\frac{1}{2}\right)} .
\end{aligned}
$$

Thus, $f_{j}$ is not merely holomorphic in $S_{\beta}$, it belongs to weighted Bergman space $A_{j}^{2}=$ $A^{2}\left(S_{\beta}, \omega_{j}(y)\right) d A$. Since $\omega_{j}$ is bounded above and below by positive constant, $A_{j}^{2}$ coincides with the unweighted Bergman space of $S_{\beta}$.

In order to obtain the expression of Szegö kernel we wish to compute the reproducing kernel for $A_{j}^{2}$. 
2.2. The weighted Bergman space of the strip. We first prove a Paley-Wiener type theorem for $A_{j}^{2}$.

Theorem 2.1. (1) Let $\omega_{j}$ be as in (5). Then

$$
\begin{aligned}
\widehat{\omega}_{j}(-2 i \xi)=2 \pi\left(\beta-\frac{\pi}{2}\right) \cosh (\pi \xi) \int_{-1}^{1} e^{-\left(\beta-\frac{\pi}{2}\right)(2 \xi-(j+1)) s} \sqrt{1+4 e^{-\left(\beta-\frac{\pi}{2}\right) s}} d s & \\
& +4 \pi \cosh \left[\left(\beta-\frac{\pi}{2}\right)\left(2 \xi-\left(j+\frac{1}{2}\right)\right] \frac{\sinh (\pi \xi)}{\xi} .\right.
\end{aligned}
$$

(2) Let $f \in A_{j}^{2}$. Then $f_{y}=f(\cdot+i y) \in L^{2}(\mathbb{R})$ for a.e. $y \in(-\beta, \beta), \widehat{f}_{y}(\xi)=e^{-y \xi} \widehat{f}_{0}(\xi)$, $\widehat{f}_{0} \in L^{2}(\mathbb{R}, \nu(\xi, j) d \xi)$ and

$$
\|f\|_{A_{j}^{2}}^{2}=\int_{\mathbb{R}}\left|\widehat{f}_{0}(\xi)\right|^{2} \nu(\xi, j) d \xi
$$

where $\nu(\xi, j)=\frac{1}{2 \pi} \widehat{\omega}_{j}(-2 i \xi)$.

(3) Conversely, let $\omega_{j}$ be as in (5) and let $\nu(\xi, j)=\frac{1}{2 \pi} \widehat{\omega}_{j}(-2 i \xi)$. Then, given $g \in L^{2}(\mathbb{R}, \nu(\xi, j) d \xi)$ for $z \in S_{\beta}$ we define

$$
f(z)=\frac{1}{2 \pi} \int_{\mathbb{R}} e^{i z \xi} g(\xi) d \xi .
$$

Then $f \in A_{j}^{2}, \widehat{f}_{0}=g$ and (7) holds.

Proof. (1) This is an elementary calculation. Decomposing $\omega_{j}$ as in (5) as sum of four terms we set

$$
\frac{1}{2 \pi} \widehat{\omega}_{j}(-2 i \xi)=\frac{1}{2 \pi} \int_{-\beta}^{\beta} e^{-2 y \xi} \omega_{j}(y) d y=I_{1}+\cdots+I_{4}
$$

Then,

$$
\begin{aligned}
I_{1}+I_{3} & =\frac{1}{2} \int_{-\beta+\pi}^{\beta} e^{-2 y \xi} e^{\left(y-\frac{\pi}{2}\right)(j+1)} \sqrt{1+4 e^{-\left(y-\frac{\pi}{2}\right)}} d y+\frac{1}{2} \int_{-\beta}^{\beta-\pi} e^{-2 y \xi} e^{\left(y+\frac{\pi}{2}\right)(j+1)} \sqrt{1+4 e^{-\left(y+\frac{\pi}{2}\right)}} d y \\
& =\cosh (\pi \xi) \int_{-\beta+\frac{\pi}{2}}^{\beta-\frac{\pi}{2}} e^{-2 t \xi} e^{t(j+1)} \sqrt{1+4 e^{-t}} d t \\
& =\cosh (\pi \xi) \int_{-1}^{1} e^{-\left(\beta-\frac{\pi}{2}\right)(2 \xi-(j+1)) s} \sqrt{1+4 e^{-\left(\beta-\frac{\pi}{2}\right) s}} d s,
\end{aligned}
$$

and

$$
\begin{aligned}
I_{2}+I_{4} & =e^{\left(\beta-\frac{\pi}{2}\right)\left(j+\frac{1}{2}\right)} \int_{\beta-\pi}^{\beta} e^{-2 y \xi} d y+e^{-\left(\beta-\frac{\pi}{2}\right)\left(j+\frac{1}{2}\right)} \int_{-\beta}^{-\beta+\pi} e^{-2 y \xi} d y \\
& =e^{-\left(\beta-\frac{\pi}{2}\right)\left(2 \xi-\left(j+\frac{1}{2}\right)\right.} \frac{\sinh (\pi \xi)}{\xi}+e^{\left(\beta-\frac{\pi}{2}\right)\left(2 \xi-\left(j+\frac{1}{2}\right)\right)} \frac{\sinh (\pi \xi)}{\xi} \\
& =2 \cosh \left[\left(\beta-\frac{\pi}{2}\right)\left(2 \xi-\left(j+\frac{1}{2}\right)\right)\right] \frac{\sinh (\pi \xi)}{\xi} .
\end{aligned}
$$

This proves (1). 
(2) We have already observed that $f \in A_{j}^{2}$ if and only if $f \in A^{2}\left(S_{\beta}\right)$. Then $f_{y}=f(\cdot+i y) \in$ $L^{2}(\mathbb{R})$ for a.e. $y \in(-\beta, \beta), \widehat{f}_{y}(\xi)=e^{-y \xi} \widehat{f}_{0}(\xi)$, see [7]. Therefore,

$$
\begin{aligned}
\|f\|_{A_{j}^{2}}^{2} & =\frac{1}{2 \pi} \int_{-\beta}^{\beta} \int_{\mathbb{R}}\left|\widehat{f}_{0}(\xi)\right|^{2} e^{-2 y \xi} d \xi \omega(y) d y \\
& =\frac{1}{2 \pi} \int_{\mathbb{R}}\left|\widehat{f}_{0}(\xi)\right|^{2} \int_{-\beta}^{\beta} e^{-2 y \xi} \omega(y) d y d \xi \\
& =\frac{1}{2 \pi} \int_{\mathbb{R}}\left|\widehat{f}_{0}(\xi)\right|^{2} \int_{-\beta}^{\beta} e^{-2 y \xi} \omega(y) d y d \xi=\int_{\mathbb{R}}\left|\widehat{f}_{0}(\xi)\right|^{2} \nu(\xi, j) d \xi .
\end{aligned}
$$

(3) Let $g$ be continuous with compact support in $\mathbb{R}$, so that $g \in L^{2}(\mathbb{R}, \nu(\xi, j) d \xi) \cap L^{2}\left(\mathbb{R}, \frac{\sinh (2 \beta \xi)}{\xi} d \xi\right)$. Then, by [7], if $f$ is defined as in (8) then $f$ is in $A^{2}\left(S_{\beta}\right)$, hence in $A_{j}^{2}$. Thus, part (2) applies and therefore $\widehat{f}_{0}=g$ and (17) holds. Since this holds true on a dense subset of $L^{2}(\mathbb{R}, \nu(\xi, j) d \xi)$ the conclusion follows.

Corollary 2.2. The reproducing kernel of $A_{j}^{2}$ is given by

$$
k_{j}\left(z_{1}, w_{1}\right)=\frac{1}{2 \pi} \int_{\mathbb{R}} \frac{e^{i\left(z_{1}-\bar{w}_{1}\right) \xi}}{\nu(\xi, j)} d \xi,
$$

where

$$
\nu(\xi, j)=\frac{1}{2 \pi} \widehat{\omega}_{j}(-2 i \xi),
$$

and $\widehat{\omega}_{j}(-2 i \xi)$ is given by (6) .

Notice that $\nu(\cdot, j)$ is even, positive and $1 / \nu(\cdot, j)$ is a Schwartz function such that $1 / \nu(\xi, j) \leq$ $c e^{-2 \beta|\xi|}$ for a positive constant $c$, independent of $j$, so that $k_{j}$ is well defined for $z_{1}, w_{1} \in S_{\beta}$.

Proof. Writing $k_{w_{1}}=k_{j}\left(\cdot, w_{1}\right)$, where $w_{1} \in S_{\beta}$, for $f \in A_{j}^{2}$ we have

$$
f\left(w_{1}\right)=\left\langle f, k_{w_{1}}\right\rangle_{A_{j}^{2}}=\left\langle\widehat{f_{0}}, \widehat{k_{w_{1}, 0}}\right\rangle_{L^{2}(\nu(\xi, j) d \xi)},
$$

and also

$$
f\left(w_{1}\right)=\frac{1}{2 \pi} \int_{\mathbb{R}} e^{i w_{1} \xi} \widehat{f}_{0}(\xi) d \xi .
$$

Therefore, $\widehat{k_{w_{1}, 0}}(\xi)=\nu(\xi, j)^{-1} e^{\bar{w}_{1} \xi}$, that is, (9) holds.

Proof of Theorem 1. This follows at once from the orthogonal decomposition $H^{2}=\bigoplus_{j \in \mathbb{Z}} \mathscr{H}_{j}$, where each $\mathscr{H}_{j}$ is isometrically equivalent to $A_{j}^{2}$ via the correspondence $\mathscr{H}_{j} \ni f_{j}\left(z_{1}\right) z_{2}^{j} \mapsto f_{j} \in A_{j}^{2}$, and Corollary 2.2 . 


\section{3. $L^{p}$-Boundedness of the SzegÖ PROJECTION}

The Szegö projection is the Hilbert space orthogonal projection of $L^{2}\left(b D_{\beta}^{\prime}\right)$ onto the closed subspace consisting of the boundary values of functions in $H^{2}$. By standard Hilbert space theory, the Szegö projection of $\varphi \in L^{2}\left(b D_{\beta}^{\prime}\right)$, is given by

$$
P \varphi(\kappa)=\int_{b D_{\beta}^{\prime}} \varphi\left(\kappa^{\prime}\right) K\left(\kappa, \kappa^{\prime}\right) d \sigma\left(\kappa^{\prime}\right),
$$

with $\kappa \in b D_{\beta}^{\prime}$.

We decompose $P$ writing

$$
P=\left(\chi_{E_{1}}+\cdots+\chi_{E_{4}}\right) P\left(\chi_{E_{1}}+\cdots+\chi_{E_{4}}\right)=\sum_{\ell, \ell^{\prime}=1}^{4} \chi_{E_{\ell}} P \chi_{E_{\ell^{\prime}}}
$$

and set, for $\ell, \ell^{\prime}=1, \ldots, 4$,

$$
P_{\ell, \ell^{\prime}}=\chi_{E_{\ell}} P \chi_{E_{\ell^{\prime}}} .
$$

Thus, it suffices to study the boundedness of the operators $P_{\ell, \ell^{\prime}}$, for $\ell, \ell^{\prime}=1, \ldots, 4$. We introduce the (global) coordinates on $E_{1}, \ldots, E_{4}$ in order to write down the operators $P_{\ell, \ell^{\prime}}$ explicitly. We set

$$
I_{1}=(-\beta+\pi, \beta), \quad I_{2}=(\beta-\pi, \beta), \quad I_{3}=(-\beta, \beta-\pi), \quad I_{4}=(-\beta,-\beta+\pi),
$$

and

$$
\mathcal{X}_{\ell}=\mathbb{R} \times I_{\ell} \times \mathbb{T}, \quad \ell=1, \ldots, 4 .
$$

Here and in what follows, we denote by $\mathbb{T}$ the 1 -dimensional torus $\mathbb{R} / 2 \pi \mathbb{Z}$.

The $L^{p}$-boundedness of the Szegö projection on $D_{\beta}^{\prime}$ is a consequence of the following theorem.

Theorem 3.1. For all $\ell, \ell^{\prime} \in\{1, \ldots, 4\}$ the following holds true.

(1) There exists an operator $T_{\ell, \ell^{\prime}}$ such that, for $1<p<\infty, P_{\ell, \ell^{\prime}}: L^{p}\left(E_{\ell^{\prime}}\right) \rightarrow L^{p}\left(E_{\ell}\right)$ is bounded if and only $T_{\ell, \ell^{\prime}}: L^{p}\left(\mathcal{X}_{\ell^{\prime}}\right) \rightarrow L^{p}\left(\mathcal{X}_{\ell}\right)$ is bounded.

(2) There exists a Fourier multiplier operator $M_{(y, t)}^{\ell, \ell^{\prime}}$ on $\mathbb{R} \times \mathbb{T}$ depending on the parameters $t \in I_{\ell^{\prime}}$ and $y \in I_{\ell}$ such that

$$
T_{\ell, \ell^{\prime}}=\int_{I_{\ell^{\prime}}} M_{(y, t)}^{\ell, \ell^{\prime}} d t
$$

(3) The operator

$$
T_{\ell, \ell^{\prime}}: L^{p}\left(\mathcal{X}_{\ell^{\prime}}\right) \rightarrow L^{p}\left(\mathcal{X}_{\ell}\right)
$$

is bounded for $1<p<\infty$.

Proof. (1) This step simply consists of writing the operators $P_{\ell, \ell^{\prime}}$ in coordinates and simplifying the innocuous weight factor. 
For $\ell=1, \ldots, 4$ and $\varphi \in L^{p}\left(E_{\ell}\right)$ we define

$$
\begin{aligned}
& \Lambda_{1} \varphi(x, y, \theta)=\frac{1}{\sqrt{1+4 e^{-\left(y-\frac{\pi}{2}\right)}}} \varphi\left(x+i y, e^{\frac{1}{2}\left(y-\frac{\pi}{2}\right)} e^{i \theta}\right), \text { for }(x, y, \theta) \in \mathcal{X}_{1} \\
& \Lambda_{2} \varphi(x, y, \theta)=e^{-\frac{1}{2}\left(\beta-\frac{\pi}{2}\right)} \varphi\left(x+i y, e^{\frac{1}{2}\left(\beta-\frac{\pi}{2}\right)} e^{i \theta}\right), \text { for }(x, y, \theta) \in \mathcal{X}_{2} ; \\
& \Lambda_{3} \varphi(x, y, \theta)=\frac{1}{\sqrt{1+4 e^{-\left(y+\frac{\pi}{2}\right)}}} \varphi\left(x+i y, e^{\frac{1}{2}\left(y+\frac{\pi}{2}\right)} e^{i \theta}\right), \text { for }(x, y, \theta) \in \mathcal{X}_{3} \\
& \Lambda_{4} \varphi(x, y, \theta)=e^{\frac{1}{2}\left(\beta-\frac{1}{2}\right)} \varphi\left(x+i y, e^{-\frac{1}{2}\left(\beta-\frac{\pi}{2}\right)} e^{i \theta}\right), \text { for }(x, y, \theta) \in \mathcal{X}_{4} .
\end{aligned}
$$

Notice that $\Lambda_{\ell}: L^{p}\left(E_{\ell}\right) \rightarrow L^{p}\left(\mathcal{X}_{\ell}\right)$ is an onto isomorphism. Hence, $P_{\ell, \ell^{\prime}}: L^{p}\left(E_{\ell}\right) \rightarrow L^{p}\left(E_{\ell^{\prime}}\right)$ is bounded if and only if $\Lambda_{\ell} P_{\ell, \ell^{\prime}} \Lambda_{\ell^{\prime}}^{-1}: L^{p}\left(\mathcal{X}_{\ell^{\prime}}\right) \rightarrow L^{p}\left(\mathcal{X}_{\ell}\right)$ is bounded.

We set

$$
T_{\ell, \ell^{\prime}}=\Lambda_{\ell} P_{\ell, \ell^{\prime}} \Lambda_{\ell^{\prime}}^{-1} \text {. }
$$

(2) We begin with $T_{1,1}$. We denote by $C_{c}\left(\mathcal{X}_{\ell}\right)$ the continuous functions with compact support in $\mathcal{X}_{\ell}$. Observe that, for $\psi \in C_{c}\left(\mathcal{X}_{1}\right), T_{1,1}$ is given by

$$
\begin{aligned}
T_{1,1} \psi(x, y, \theta) & =\int_{0}^{2 \pi} \int_{-\beta+\pi}^{\beta} \int_{\mathbb{R}} \psi\left(s, t, \theta^{\prime}\right) K\left(\left(x+i y, e^{\frac{1}{2}\left(y-\frac{\pi}{2}\right)} e^{i \theta}\right),\left(s+i t, e^{\frac{1}{2}\left(t-\frac{\pi}{2}\right)} e^{i \theta^{\prime}}\right)\right) d s d t d \theta^{\prime} \\
& =\int_{0}^{2 \pi} \int_{-\beta+\pi}^{\beta} \int_{\mathbb{R}} \psi\left(s, t, \theta^{\prime}\right)\left(\sum_{j \in \mathbb{Z}} e^{(t+y-\pi) \frac{j}{2}} e^{i j\left(\theta-\theta^{\prime}\right)} \frac{1}{2 \pi} \int_{\mathbb{R}} \frac{e^{i(x-s) \xi-(t+y) \xi}}{\nu(\xi, j)} d \xi\right) d s d t d \theta^{\prime} \\
& =: \int_{I_{1}} \int_{\mathbb{T}} \int_{\mathbb{R}} \psi\left(s, t, \theta^{\prime}\right) H\left(x-s, y+t, \theta-\theta^{\prime}\right) d s d \theta^{\prime} d t .
\end{aligned}
$$

Thus, $T_{1,1}$ is the composition of a convolution operator on $\mathbb{R} \times \mathbb{T}$ in the first and third variables, depending on the parameters $(y, t)$ with an integration in the $t$ variable:

$$
T_{1,1} \psi(x, y, \theta)=\int_{I_{1}} M_{(y, t)}^{1,1}(\psi(\cdot, t, \cdot))(x, \theta) d t,
$$

where $M_{(y, t)}^{1,1}$ is the convolution operator on $\mathbb{R} \times \mathbb{T}$ with kernel $H(x, y+t, \theta)$. Notice that the Fourier multiplier associated to $M_{(y, t)}^{1,1}$, that is, the Fourier transform of this kernel on $\mathbb{R} \times \mathbb{T}$, is

$$
\begin{aligned}
\frac{e^{(t+y-\pi) \frac{j}{2}} e^{-(t+y) \xi}}{\nu(\xi, j)} & =e^{-\frac{1}{2}(t+y-\pi)} \frac{e^{\pi \xi} e^{-\frac{1}{2}(t+y-\pi)(2 \xi-(j+1))}}{\nu(\xi, j)} \\
& =: e^{-\frac{1}{2}(t+y-\pi)} m_{(y, t)}^{1,1}(\xi, j)
\end{aligned}
$$

where $t, y \in I_{1}$.

Remark. We point out that, for the later part of our proof, it is convenient to express the multiplier as function of $\xi$ and $2 \xi-(j+1)$, since the term $\nu(\xi, j)$ is in fact already function of such variables. We will keep this approach in the expression of all the multipliers $m_{(y, t)}^{\ell, \ell^{\prime}}$ below. 
The operator $T_{3,3}$ is quite similar to $T_{1,1}$, and arguing in the same way we obtain that

$$
\begin{aligned}
T_{3,3} \psi(x, y, \theta) & =\int_{0}^{2 \pi} \int_{-\beta}^{\beta-\pi} \int_{\mathbb{R}} \psi\left(s, t, \theta^{\prime}\right) K\left(\left(x+i y, e^{\frac{1}{2}\left(y+\frac{\pi}{2}\right)} e^{i \theta}\right),\left(s+i t, e^{\frac{1}{2}\left(t+\frac{\pi}{2}\right)} e^{i \theta^{\prime}}\right)\right) d s d t d \theta^{\prime} \\
& =\int_{0}^{2 \pi} \int_{-\beta}^{\beta-\pi} \int_{\mathbb{R}} \psi\left(s, t, \theta^{\prime}\right)\left(\sum_{j \in \mathbb{Z}} e^{(t+y+\pi) \frac{j}{2}} e^{i j\left(\theta-\theta^{\prime}\right)} \frac{1}{2 \pi} \int_{\mathbb{R}} \frac{e^{i(x-s) \xi-(t+y) \xi}}{\nu(\xi, j)} d \xi\right) d s d t d \theta^{\prime} \\
& =\int_{I_{3}} M_{(y, t)}^{3,3}(\psi(\cdot, t, \cdot))(x, \theta) d t
\end{aligned}
$$

where in this case $M_{(y, t)}^{3,3}$ is the convolution operator whose associate multiplier on $\mathbb{R} \times \mathbb{T}$ is

$$
\begin{aligned}
\frac{e^{(t+y+\pi) \frac{j}{2}} e^{-(t+y) \xi}}{\nu(\xi, j)} & =e^{-\frac{1}{2}(t+y+\pi)} \frac{e^{\pi \xi} e^{-\frac{1}{2}(t+y+\pi)(2 \xi-(j+1))}}{\nu(\xi, j)} \\
& =: e^{-\frac{1}{2}(t+y+\pi)} m_{(y, t)}^{3,3}(\xi, j)
\end{aligned}
$$

with $t, y \in I_{3}$.

Next we turn to the case of $T_{2,2,}$. For $\psi \in C_{c}\left(\mathcal{X}_{2}\right)$ we have that

$$
\begin{aligned}
T_{2,2} \psi(x, y, \theta) & =\int_{0}^{2 \pi} \int_{\beta-\pi}^{\beta} \int_{\mathbb{R}} \psi\left(s, t, \theta^{\prime}\right) K\left(\left(x+i y, e^{\frac{1}{2}\left(\beta-\frac{\pi}{2}\right)} e^{i \theta}\right),\left(s+i t, e^{\frac{1}{2}\left(\beta-\frac{\pi}{2}\right)} e^{i \theta^{\prime}}\right)\right) d s d t d \theta^{\prime} \\
& =\int_{0}^{2 \pi} \int_{\beta-\pi}^{\beta} \int_{\mathbb{R}} \psi\left(s, t, \theta^{\prime}\right)\left(\sum_{j \in \mathbb{Z}} e^{\left(\beta-\frac{\pi}{2}\right) j} e^{i j\left(\theta-\theta^{\prime}\right)} \frac{1}{2 \pi} \int_{\mathbb{R}} \frac{e^{i(x-s) \xi-(t+y) \xi}}{\nu(\xi, j)} d \xi\right) d s d t d \theta^{\prime} \\
& =\int_{I_{2}} M_{(y, t)}^{2,2}(\psi(\cdot, t, \cdot))(x, \theta) d t
\end{aligned}
$$

where in this case the associated multiplier is

$$
\begin{aligned}
\frac{e^{\left(\beta-\frac{\pi}{2}\right) j} e^{-(t+y) \xi}}{\nu(\xi, j)} & =e^{-\left(\beta-\frac{\pi}{2}\right)} \frac{e^{-\left(\beta-\frac{\pi}{2}\right)(2 \xi-(j+1))} e^{(2 \beta-\pi-(t+y)) \xi}}{\nu(\xi, j)} \\
& =: e^{-\left(\beta-\frac{\pi}{2}\right)} m_{(y, t)}^{2,2}(\xi, j)
\end{aligned}
$$

with $t, y \in I_{2}$.

The case of $T_{4,4}$, is again quite similar. We obtain that, for $\psi$ defined on $I_{4}$,

$$
T_{4,4} \psi(x, y, \theta)=\int_{I_{4}} M_{(y, t)}^{4,4}(\psi(\cdot, t, \cdot))(x, \theta) d t,
$$

where the Fourier multiplier of $M_{(y, t)}^{4,4}$ is

$$
\begin{aligned}
\frac{e^{-\left(\beta-\frac{\pi}{2}\right) j} e^{-(t+y) \xi}}{\nu(\xi, j)} & =e^{\left(\beta-\frac{\pi}{2}\right)} \frac{e^{\left(\beta-\frac{\pi}{2}\right)(2 \xi-(j+1))} e^{-(2 \beta-\pi+t+y) \xi}}{\nu(\xi, j)} \\
& =: e^{\left(\beta-\frac{\pi}{2}\right)} m_{(y, t)}^{4,4}(\xi, j)
\end{aligned}
$$

with $t, y \in I_{4}$.

Next we turn to the non-diagonal cases, that is, the operators $T_{\ell, \ell^{\prime}}$ with $\ell \neq \ell^{\prime}$. We proceed in the same fashion as in the previous cases. 
We now describe $T_{2,1}$. For $\psi$ sufficiently regular and defined on $\mathcal{X}_{1}$ and $(x, y, \theta) \in \mathcal{X}_{2}$ we have

$$
\begin{aligned}
T_{2,1} \psi(x, y, \theta) & =\int_{0}^{2 \pi} \int_{-\beta+\pi}^{\beta} \int_{\mathbb{R}} \psi\left(s, t, \theta^{\prime}\right) K\left(\left(x+i y, e^{\frac{1}{2}\left(\beta-\frac{\pi}{2}\right)} e^{i \theta}\right),\left(s+i t, e^{\frac{1}{2}\left(t-\frac{\pi}{2}\right)} e^{i \theta^{\prime}}\right)\right) d s d t d \theta^{\prime} \\
& =\int_{0}^{2 \pi} \int_{-\beta+\pi}^{\beta} \int_{\mathbb{R}} \psi\left(s, t, \theta^{\prime}\right)\left(\sum_{j \in \mathbb{Z}} e^{(\beta-\pi+t) \frac{j}{2}} e^{i j\left(\theta-\theta^{\prime}\right)} \frac{1}{2 \pi} \int_{\mathbb{R}} \frac{e^{i(x-s) \xi-(t+y) \xi}}{\nu(\xi, j)} d \xi\right) d s d t d \theta^{\prime} \\
& =\int_{I_{1}} M_{(y, t)}^{2,1}(\psi(\cdot, t, \cdot))(x, \theta) d t
\end{aligned}
$$

where $M_{(y, t)}^{2,1}$ is a convolution operator on $\mathbb{R} \times \mathbb{T}$ whose associated multiplier on $\mathbb{R} \times \mathbb{T}$ is

$$
\begin{aligned}
\frac{e^{(\beta-\pi+t) \frac{j}{2}} e^{-(t+y) \xi}}{\nu(\xi, j)} & =e^{-\frac{1}{2}(\beta-\pi+t)} \frac{e^{-\frac{1}{2}(\beta+t-\pi)(2 \xi-(j+1))} e^{-(y-\beta+\pi) \xi}}{\nu(\xi, j)} \\
& =: e^{-\frac{1}{2}(\beta-\pi+t)} m_{(y, t)}^{2,1}(\xi, j)
\end{aligned}
$$

where $t \in I_{1}$ and $y \in I_{2}$.

Next we focus on the operator $T_{3,1}$. We point out that the Fourier multiplier we obtain for this operator has a better decay of the multipliers that appeared so far. This is due to the fact that $\operatorname{dist}\left(\mathcal{X}_{3}, \mathcal{X}_{1}\right)>0$. For $\psi$ sufficiently regular and defined on $\mathcal{X}_{1}$ and $(x, y, \theta) \in \mathcal{X}_{3}$ we have

$$
\begin{aligned}
T_{3,1} \psi(x, y, \theta) & =\int_{0}^{2 \pi} \int_{-\beta+\pi}^{\beta} \int_{\mathbb{R}} \psi\left(s, t, \theta^{\prime}\right) K\left(\left(x+i y, e^{\frac{1}{2}\left(y+\frac{\pi}{2}\right)} e^{i \theta}\right),\left(s+i t, e^{\frac{1}{2}\left(t-\frac{\pi}{2}\right)} e^{i \theta^{\prime}}\right)\right) d s d t d \theta^{\prime} \\
& =\int_{0}^{2 \pi} \int_{-\beta+\pi}^{\beta} \int_{\mathbb{R}} \psi\left(s, t, \theta^{\prime}\right)\left(\sum_{j \in \mathbb{Z}} e^{(y+t) \frac{j}{2}} e^{i j\left(\theta-\theta^{\prime}\right)} \frac{1}{2 \pi} \int_{\mathbb{R}} \frac{e^{i(x-s) \xi-(t+y) \xi}}{\nu(\xi, j)} d \xi\right) d s d t d \theta^{\prime} \\
& =\int_{I_{1}} M_{(y, t)}^{3,1}(\psi(\cdot, t, \cdot))(x, \theta) d t
\end{aligned}
$$

where the Fourier transform on $\mathbb{R} \times \mathbb{T}$ of the kernel of the convolution operator $M_{(y, t)}^{3,1}$ is

$$
\begin{aligned}
\frac{e^{(\beta-\pi+t) \frac{j}{2}} e^{-(t+y) \xi}}{\nu(\xi, j)} & =e^{-\frac{1}{2}(y+t)} \frac{e^{-\frac{1}{2}(y+t)(2 \xi-(j+1))}}{\nu(\xi, j)} \\
& =: e^{-\frac{1}{2}(y+t)} m_{(y, t)}^{3,1}(\xi, j),
\end{aligned}
$$

where $t \in I_{1}$ and $y \in I_{3}$.

In a similar way we obtain the operator $T_{4,1}$. For a sufficiently regular function $\psi$ defined on $\mathcal{X}_{4}$, we obtain

$$
T_{4,1} \psi(x, y, \theta)=\int_{I_{1}} M_{(y, t)}^{4,1}(\psi(\cdot, t, \cdot))(x, \theta) d t
$$

where the Fourier multiplier of $M_{(y, t)}^{4,1}$ is

$$
\begin{aligned}
\frac{e^{-(\beta-t) \frac{j}{2}} e^{-(t+y) \xi}}{\nu(\xi, j)} & =e^{\frac{1}{2}(\beta-t)} \frac{e^{\frac{1}{2}(\beta-t)(2 \xi-(j+1))} e^{-(\beta+y) \xi}}{\nu(\xi, j)} \\
& =: e^{\frac{1}{2}(\beta-t)} m_{(y, t)}^{4,1}(\xi, j),
\end{aligned}
$$


where $y \in I_{4}$ and $t \in I_{1}$.

All other operators $T_{\ell, \ell^{\prime}}$ can be obtained by slight modification of the arguments above.

If $\psi$ is a suitable function defined on $\mathcal{X}_{\ell^{\prime}}$ and $(x, y, \theta) \in \mathcal{X}_{\ell}$, then a generic operator $T_{\ell, \ell^{\prime}}$ turns out to be of the form

$$
T_{\ell, \ell^{\prime}} \psi(x, y, \theta)=\int_{I_{\ell^{\prime}}} M_{(y, t)}^{\ell, \ell^{\prime}}(\psi(\cdot, t, \cdot))(x, \theta) d t
$$

where $M_{(y, t)}^{\ell, \ell^{\prime}}$ is a Fourier multiplier operator with multiplier of the form

$$
\alpha^{\ell, \ell^{\prime}}(y, t) m_{(y, t)}^{\ell, \ell^{\prime}}(\xi, j)
$$

where $(y, t) \in I_{\ell} \times I_{\ell^{\prime}}$ and the factor $\alpha^{\ell, \ell^{\prime}}$ is a positive function which is bounded together with all its derivatives. Therefore, in order to prove the $L^{p}$ boundedness, it suffices to consider the multipliers $m_{(y, t)}^{\ell, \ell^{\prime}}$. It is now easy to see that

$$
\begin{aligned}
& m_{(y, t)}^{1,2}(\xi, j)=\frac{e^{-\frac{1}{2}(\beta-\pi+y)(2 \xi-(j+1))} e^{-(t-\beta+\pi) \xi}}{\nu(\xi, j)}, \quad m_{(y, t)}^{1,4}(\xi, j)=\frac{e^{\frac{1}{2}(\beta-y)(2 \xi-(j+1))} e^{-(\beta+t) \xi}}{\nu(\xi, j)} \\
& m_{(y, t)}^{3,2}(\xi, j)=\frac{e^{-\frac{1}{2}(\beta+y)(2 \xi-(j+1))} e^{(\beta-t) \xi}}{\nu(\xi, j)}, \quad m_{(y, t)}^{2,3}(\xi, j)=\frac{e^{\frac{1}{2}(\beta+t)(2 \xi-(j+1))} e^{(\beta-y) \xi}}{\nu(\xi, j)}, \\
& m_{(y, t)}^{3,4}(\xi, j)=\frac{e^{\frac{1}{2}(\beta-\pi-y)(2 \xi-(j+1))} e^{-(\beta-\pi+t) \xi}}{\nu(\xi, j)}, \quad m_{(y, t)}^{4,3}(\xi, j)=\frac{e^{\frac{1}{2}(\beta-\pi-t)(2 \xi-(j+1))} e^{-(\beta-\pi+y) \xi}}{\nu(\xi, j)} \\
& m_{(y, t)}^{1,3}(\xi, j)=\frac{e^{-\frac{1}{2}(t+y)(2 \xi-(j+1))}}{\nu(\xi, j)}, \quad m_{(y, t)}^{4,2}(\xi, j)=\frac{e^{(y+t) \xi}}{\nu(\xi, j)}, \quad m_{(y, t)}^{2,4}(\xi, j)=\frac{e^{-(y+t) \xi}}{\nu(\xi, j)} .
\end{aligned}
$$

(3) We proceed by applying Minkowski's integral inequality. Let $\ell, \ell^{\prime} \in\{1, \ldots, 4\}$. Then

$$
\left|T_{\ell, \ell^{\prime}} \psi(x, y, \theta)\right| \leq \int_{I_{\ell^{\prime}}}\left|M_{(y, t)}^{\ell, \ell^{\prime}}(\psi(\cdot, t, \cdot))(x, \theta)\right| d t
$$

so that

$$
\begin{aligned}
\int_{\mathcal{X}_{\ell}}\left|M_{(y, t)}^{\ell, \ell^{\prime}} \psi(x, y, \theta)\right|^{p} d x d y d \theta & \leq \int_{I_{\ell}} \int_{\mathbb{R} \times \mathbb{T}}\left(\int_{I_{\ell^{\prime}}}\left|M_{(y, t)}^{\ell, \ell^{\prime}}(\psi(\cdot, t, \cdot))(x, \theta)\right| d t\right)^{p} d x d \theta d y \\
& =\int_{I_{\ell}}\left\{\int_{\mathbb{R} \times \mathbb{T}}\left(\int_{I_{\ell^{\prime}}}\left|M_{(y, t)}^{\ell, \ell^{\prime}}(\psi(\cdot, t, \cdot))(x, \theta)\right| d t\right)^{p} d x d \theta\right\}^{p / p} d y \\
& \leq \int_{I_{\ell}}\left\{\int_{I_{\ell^{\prime}}}\left(\int_{\mathbb{R} \times \mathbb{T}}\left|M_{(y, t)}^{\ell, \ell^{\prime}}(\psi(\cdot, t, \cdot))(x, \theta)\right|^{p} d x d \theta\right)^{1 / p} d t\right\}^{p} d y .
\end{aligned}
$$

Then, if we denote by $N_{p}^{\ell, \ell^{\prime}}(y, t)$ the operator norm of $M_{(y, t)}^{\ell, \ell^{\prime}}$ on $L^{p}(\mathbb{R} \times \mathbb{T})$ we have

$$
\begin{aligned}
\left\|T_{\ell, \ell^{\prime}} \psi\right\|_{L^{p}\left(\mathcal{X}_{\ell}\right)}^{p} & =\int_{\mathcal{X}_{\ell}}\left|T_{\ell, \ell^{\prime}} \psi(x, y, \theta)\right|^{p} d x d y d \theta \\
& \leq \int_{I_{\ell}}\left\{\int_{I_{\ell^{\prime}}} N_{p}^{\ell, \ell^{\prime}}(y, t)\|\psi(\cdot, t, \cdot)\|_{L^{p}(\mathbb{R} \times \mathbb{T})} d t\right\}^{p} d y .
\end{aligned}
$$

Thus, we have reduced ourselves to showing that: 
(A) the operator $M_{(y, t)}^{\ell, \ell^{\prime}}$ is bounded on $L^{p}(\mathbb{R} \times \mathbb{T})$ for a.a. $t \in I_{\ell^{\prime}}$ and $y \in I_{\ell}$ with norm $N_{p}^{\ell, \ell^{\prime}}(y, t)$

(B) the integral operator $\mathcal{N}_{\ell, \ell^{\prime}}$ with integral kernel $N_{p}^{\ell, \ell^{\prime}}(y, t)$, is bounded $\mathcal{N}_{\ell, \ell^{\prime}}: L^{p}\left(I_{\ell^{\prime}}\right) \rightarrow$ $L^{p}\left(I_{\ell}\right)$, for $1<p<\infty$.

¿From these two facts, the boundedness of $T_{\ell, \ell^{\prime}}$, hence the one of $P$, follows at once.

We point that the integral operator $\mathcal{N}_{\ell, \ell^{\prime}}$ with integral kernel $N_{p}^{\ell, \ell^{\prime}}(y, t)$ turns to be an operator of Hilbert type (see [22]). This fact is not surprising as several operators in several complex variables appear as composition of singular integrals (as the operator $M_{(y, t)}^{\ell, \ell^{\prime}}$ ) and operators of Hilbert type.

In order to prove $(\mathbf{A})$, recall that $M_{(y, t)}^{\ell, \ell^{\prime}}$ is a Fourier multiplier operator on $\mathbb{R} \times \mathbb{Z}$, whose multiplier is given in the identities (16-22) and (24). By the boundedness of the factors appearing on the right hand sides of such equations, it suffices to study the boundedness of the multipliers $m_{(y, t)}^{\ell, \ell^{\prime}}(\xi, j)$, for $\ell, \ell^{\prime} \in\{1, \ldots, 4\}$.

It suffices to show that $m_{(y, t)}^{\ell, \ell^{\prime}}(\xi, \eta)$ is a bounded Fourier multiplier on $L^{p}\left(\mathbb{R}^{2}\right)$, see [6, Thm. 3.6.7]. By the affine change of variables

$$
\left\{\begin{array}{l}
\xi^{\prime}=\pi \xi \\
\eta^{\prime}=\left(\beta-\frac{\pi}{2}\right)(2 \xi-(\eta+1)),
\end{array}\right.
$$

it suffices to show that the functions $\tilde{m}_{(y, t)}^{\ell, \ell^{\prime}}\left(\xi^{\prime}, \eta^{\prime}\right)$ give rise to bounded Fourier multipliers on $L^{p}\left(\mathbb{R}^{2}\right)$.

Denote by $T_{m}$ the Fourier multiplier operator with multiplier $m$. Then, using [6, Thm. 3.6.7] again, we have the bounds

$$
\left\|M_{(y, t)}^{\ell, \ell^{\prime}}\right\|_{L^{p}(\mathbb{R} \times \mathbb{Z}) \rightarrow L^{p}(\mathbb{R} \times \mathbb{Z})} \leq\left\|T_{m_{(y, t)}^{\ell, \ell^{\prime}}}\right\|_{L^{p}\left(\mathbb{R}^{2}\right) \rightarrow L^{p}\left(\mathbb{R}^{2}\right)} \leq C\left\|T_{\tilde{m}_{(y, t)}^{\ell, \ell^{\prime}}}\right\|_{L^{p}\left(\mathbb{R}^{2}\right) \rightarrow L^{p}\left(\mathbb{R}^{2}\right)},
$$

with $C$ independent of $y$ and $t$.

Proof of (A). We shall show that $\tilde{m}_{(y, t)}^{\ell, \ell^{\prime}}$ is a Marcinkiewicz multiplier in $\mathbb{R}^{2}$ with norm $N_{p}^{\ell, \ell^{\prime}}(y, t)$, for $\ell, \ell^{\prime} \in\{1, \ldots, 4\}$.

We recall that $m$ is said to be a Marcinkiewicz multiplier in $\mathbb{R}^{2}$ if there exists $C>0$ such that

$$
\sup _{\xi, \eta \in \mathbb{R} \backslash\{0\}}\left|\partial_{\xi}^{k_{1}} \partial_{\eta}^{k_{2}} m(\xi, \eta)\right| \leq C \frac{1}{|\xi|^{k_{1}}|\eta|^{k_{2}}}
$$

for $k_{1}, k_{2} \geq 0, k_{1}+k_{2} \leq 2$, see e.g. [6. Corollary 5.2.5]. Thus, we wish to show that $\tilde{m}_{(y, t)}^{\ell, \ell^{\prime}}$ are Marcinkiewicz multipliers in $\mathbb{R}^{2}$ and estimates their operator norms, for $\ell, \ell^{\prime} \in\{1, \ldots, 4\}$.

In view of the change of coordinates (26) (and dropping the primes to ease notation), we set

$$
D(\xi, \eta)=\cosh \eta \frac{\sinh \xi}{\xi}+\frac{\sinh \eta}{\eta} \cosh \xi
$$

and

$$
Q(\xi, \eta)=\frac{D(\xi, \eta)}{\tilde{\nu}(\xi, \eta)}
$$

where $\tilde{\nu}(\xi, \eta)$ denotes the function $\nu(\xi, \eta)$ after the change of variables (26). 
In the diagonal cases, the multipliers $\tilde{m}_{(y, t)}^{1,1}, \ldots, \tilde{m}_{(y, t)}^{4,4}$, are given by (16-19), namely,

$$
\tilde{m}_{(y, t)}^{\ell, \ell}(\xi, \eta)=\frac{e^{\gamma \xi} e^{\alpha \eta}}{D(\xi, \eta)} \cdot Q(\xi, \eta)
$$

where

$$
\begin{array}{ll}
\text { if } \ell=1, & \alpha=\frac{\pi-(t+y)}{2 \beta-\pi}, \gamma=1, t, y \in I_{1} ; \\
\text { if } \ell=2, & \alpha=-1, \gamma=\frac{2 \beta-\pi-(t+y)}{\pi}, t, y \in I_{2} ; \\
\text { if } \ell=3, & \alpha=-\frac{t+y+\pi}{2 \beta-\pi}, \gamma=1, t, y \in I_{3} ; \\
\text { if } \ell=4, & \alpha=1, \gamma=-\frac{2 \beta-\pi+t+y}{\pi}, t, y \in I_{4} .
\end{array}
$$

Next we turn to the off-diagonal cases. As in the diagonal case, from (16-22), (24) and the change of variables (26), we obtain again

$$
\tilde{m}_{(y, t)}^{\ell, \ell^{\prime}}(\xi, \eta)=\frac{e^{\gamma \xi} e^{\alpha \eta}}{D(\xi, \eta)} \cdot Q(\xi, \eta)
$$

where now,

$$
\begin{array}{ll}
\text { if }\left(\ell, \ell^{\prime}\right)=(2,1), & \alpha=-\frac{\beta-\pi+t}{2 \beta-\pi}, \gamma=-\frac{y-\beta+\pi}{\pi}, t \in I_{1}, y \in I_{2} ; \\
\text { if }\left(\ell, \ell^{\prime}\right)=(4,1), & \alpha=-\frac{\beta-t}{2 \beta-\pi}, \gamma=-\frac{\beta+y}{\pi}, t \in I_{1}, y \in I_{4} ; \\
\text { if }\left(\ell, \ell^{\prime}\right)=(1,2), & \alpha=-\frac{\beta-\pi+y}{2 \beta-\pi}, \gamma=-\frac{t-\beta+\pi}{\pi}, t \in I_{2}, y \in I_{1} ; \\
\text { if }\left(\ell, \ell^{\prime}\right)=(3,2), & \alpha=-\frac{\beta+y}{2 \beta-\pi}, \gamma=\frac{\beta-t}{\pi}, t \in I_{2}, y \in I_{3} ; \\
\text { if }\left(\ell, \ell^{\prime}\right)=(2,3), & \alpha=\frac{\beta+t}{2 \beta-\pi}, \gamma=-\frac{\beta-y}{\pi}, t \in I_{3}, y \in I_{2} ; \\
\text { if }\left(\ell, \ell^{\prime}\right)=(4,3), & \alpha=\frac{\beta-\partial-t}{2 \beta-\pi}, \gamma=\frac{\pi-\beta-y}{\pi}, t \in I_{3}, y \in I_{4} ; \\
\text { if }\left(\ell, \ell^{\prime}\right)=(1,4), & \alpha=\frac{\beta-y}{2 \beta-\pi}, \gamma=-\frac{\beta+t}{\pi}, t \in I_{4}, y \in I_{1} ; \\
\text { if }\left(\ell, \ell^{\prime}\right)=(3,4), & \alpha=\frac{\beta-\pi-y}{2 \beta-\pi}, \gamma=\frac{\pi-\beta-t}{\pi}, t \in I_{4}, y \in I_{3} .
\end{array}
$$

We point out that the cases when $\left(\ell, \ell^{\prime}\right) \in\{(3,1),(4,2),(1,3),(2,4)\}$ are easier and the multipliers have a better decay. This is due to the fact that, as we already mentioned, $\operatorname{dist}\left(\mathcal{X}_{1}, \mathcal{X}_{3}\right)$, $\operatorname{dist}\left(\mathcal{X}_{2}, \mathcal{X}_{4}\right)>0$. In these special cases we have

$$
\tilde{m}_{(y, t)}^{\ell, \ell^{\prime}}(\xi, \eta)=\frac{e^{\gamma \xi}}{D(\xi, \eta)} \cdot Q(\xi, \eta),
$$


where

$$
\begin{array}{ll}
\text { if }\left(\ell, \ell^{\prime}\right)=(3,1), & \gamma=-\frac{t+y}{2 \beta-\pi}, t \in I_{1}, y \in I_{3} . \\
\text { if }\left(\ell, \ell^{\prime}\right)=(4,2), & \gamma=\frac{t+y}{\pi}, t \in I_{2}, y \in I_{4} . \\
\text { if }\left(\ell, \ell^{\prime}\right)=(1,3), & \gamma=-\frac{t+y}{2 \beta-\pi}, t \in I_{3}, y \in I_{1} . \\
\text { if }\left(\ell, \ell^{\prime}\right)=(2,4), & \gamma=-\frac{t+y}{\pi}, t \in I_{4}, y \in I_{3} .
\end{array}
$$

We shall make use of the following lemmas and defer their proofs to Section 4.

Lemma 3.2. The function

$$
Q(\xi, \eta)=\frac{\cosh \eta \frac{\sinh \xi}{\xi}+\frac{\sinh \eta}{\eta} \cosh \xi}{2 \pi \frac{\sinh \xi}{\xi} \cosh \left[\eta+\frac{1}{2}\left(\beta-\frac{\pi}{2}\right)\right]+\left(\beta-\frac{\pi}{2}\right) \cosh \xi\left(\int_{-1}^{1} e^{-\eta s} \sqrt{1+e^{-\left(\beta-\frac{\pi}{2}\right) s} d s}\right)}
$$

is a Marcinkiewicz multiplier in $\mathbb{R}^{2}$.

Lemma 3.3. The function

$$
m_{\alpha}(\xi, \eta)=\frac{e^{\xi} e^{\alpha \eta}}{D(\xi, \eta)}
$$

is a Marcinkiewicz multiplier in $\mathbb{R}^{2}$ for every $0<\alpha<1$. Moreover the operator norm of the associated multipliers operator is bounded by $\frac{C}{1-\alpha}$.

Lemma 3.4. The function

$$
m_{\alpha, \gamma}(\xi, \eta)=\frac{e^{\gamma \xi} e^{\alpha \eta}}{D(\xi, \eta)}
$$

is a Marcinkiewicz multiplier on $\mathbb{R}^{2}$ for every $0<\alpha, \gamma<1$. Moreover the norm of the associated multiplier operator is bounded by $\frac{C}{(1-\alpha)+(1-\gamma)}$.

Proof of (B). We now prove (B) in the case of the operator $\mathcal{N}_{1,1}$ associated to $T_{1,1}$. The remaining cases can be proved similarly with minor modifications. We show that $\mathcal{N}_{1,1}$ is a Hilbert-type integral operator and can be studied with standard technique by means of Schur's test (see, e.g., [6, Appendix I] ).

We suppose that the parameter $\alpha$ appearing in $\tilde{m}_{y, t}^{1,1}$, see (29), is positive. The case of $\alpha$ negative can be similarly treated. From (25), (29) and Lemma 3.3 we deduce

$$
\begin{aligned}
\left\|T_{1,1} \psi\right\|_{L^{p}\left(\mathcal{X}_{\ell}\right.}^{p} & \leq C \int_{I_{1}}\left\{\int_{I_{1}} \frac{1}{1-\frac{\pi-(t+y)}{2 \beta-\pi}}\|\psi(\cdot, t, \cdot)\|_{L^{p}(\mathbb{R} \times \mathbb{T})} d t\right\}^{p} d y \\
& \leq C \int_{I_{1}}\left\{\int_{I_{1}} \frac{1}{(\beta-\pi+t)+(\beta-\pi+y)}\|\psi(\cdot, t, \cdot)\|_{L^{p}(\mathbb{R} \times \mathbb{T})} d t\right\}^{p} d y .
\end{aligned}
$$

Therefore, the integral operator $\mathcal{N}_{1,1}$ associated to $T_{1,1}$ has positive integral kernel

$$
N_{p}^{1,1}(y, t)=\frac{1}{(\beta-\pi+t)+(\beta-\pi+y)} .
$$


It suffices to show that there exists a constant $C>0$ such that

$$
\int_{I_{1}} \frac{1}{(\beta-\pi+t)+(\beta-\pi+y)} \varphi^{p}(t) d t \leq C \varphi^{p}(y)
$$

and

$$
\int_{I_{1}} \frac{1}{(\beta-\pi+t)+(\beta-\pi+y)} \varphi^{q}(t) d t \leq C \varphi^{q}(y)
$$

for every $y \in I_{1}$, where $q$ is the conjugate exponent of $p$. It is enough to choose $\varphi(t)=$ $(\beta-\pi+t)^{-\frac{1}{q p}}$ and the desired estimates are easily proved; see [22, (2.2)] or [6, Appendix I.3] for details.

The estimates for the operators $T_{\ell, \ell}, \ell=2,3,4$ follow in the same fashion.

When $\ell \neq \ell$, it suffices to perform an affine change of variables so that $I_{\ell}$ coincides with $I_{\ell^{\prime}}$ and to use again Schur's test. We leave the elementary details to the reader.

\section{Proofs of Theorem 2 and Lemmas $3.2+3.4$}

4.1. Proof of Theorem 2. In order to complete the proof it suffices to show that

$$
P: W^{k, p}\left(b D_{\beta}^{\prime}\right) \rightarrow W^{k, p}\left(b D_{\beta}^{\prime}\right)
$$

is bounded when $1<p<\infty$ and $k$ is a positive integer. Clearly, again it also suffices to show that the operators $T_{\ell, \ell^{\prime}}$ in Theorem 3.1 are bounded on $W^{k, p}\left(b D_{\beta}^{\prime}\right)$, when $1<p<\infty$ and $k$ is a positive integer.

Since $T_{\ell, \ell^{\prime}}=\int_{I_{\ell^{\prime}}} M_{(y, t)}^{\ell, \ell^{\prime}} d t$, where $M_{(y, t)}^{\ell, \ell^{\prime}}$ is a Fourier multiplier operator on $\mathbb{R} \times \mathbb{T}$, derivatives in $x$ and $\theta$ commute with $T_{\ell, \ell^{\prime}}$ and therefore we have nothing to prove.

Finally, consider derivatives in $y \in I_{\ell}$. We claim that $\partial_{y}\left(T_{\ell, \ell^{\prime}} f\right)=T_{\ell, \ell^{\prime}}\left(\partial_{x} f\right)$, up to a multiplicative constant. This commutation rule will prove the theorem.

For, when we examine the dependence of the multiplier $m_{(t, y)}^{\ell, \ell^{\prime}}$ in $y$ we see that they either have no effect on the multiplier itself (if the derivative falls on a factor of the form $e^{c y}, c$ a constant), or produce a term that has the form $\xi \cdot m_{(t, y)}^{\ell, \ell^{\prime}}$ (when the derivative falls on a factor of the form $\left.e^{y \xi}\right)$. Multiplication by $\xi$ on the Fourier transform side can be viewed as a derivative in $x$ of the function the operator $T_{\ell, \ell^{\prime}}$ is acting on, and we are done.

4.2. Proof of Lemma 3.2. It is easy to see that

$$
\frac{D(\xi, \eta)}{2\left(\beta-\frac{\pi}{2}\right) \cosh \eta \frac{\sinh \eta}{\eta}+2 \pi \frac{\sinh \xi}{\xi} \cosh \eta}
$$

is a Marcinkiewicz multiplier, therefore, in order to prove that $Q(\xi, \eta)$ is a multiplier, it suffices to focus on

$$
\begin{aligned}
\frac{2\left(\beta-\frac{\pi}{2}\right) \cosh \eta \frac{\sinh \eta}{\eta}+2 \pi \frac{\sinh \xi}{\xi} \cosh \eta}{\tilde{\nu}(\xi, \eta)} & =\frac{2\left(\beta-\frac{\pi}{2}\right) \frac{\tanh \eta}{\eta}+2 \pi \frac{\tanh \xi}{\xi}}{2 \pi \frac{\tanh \xi}{\xi}+\left(\beta-\frac{\pi}{2}\right) F(\eta)} \cdot \frac{\cosh \eta}{\cosh \left(\eta+\frac{1}{2}\left(\beta-\frac{\pi}{2}\right)\right)} \\
& =: \tilde{Q}(\xi, \eta) \cdot P(\eta),
\end{aligned}
$$


where

$$
\begin{aligned}
F(\eta) & =\frac{\int_{|s|<1} e^{-\eta s} \sqrt{1+4^{-\left(\beta-\frac{\pi}{2}\right) s}} d s}{\cosh \left(\eta+\frac{1}{2}\left(\beta-\frac{\pi}{2}\right)\right)} \\
& =\frac{\int_{|s|<1} e^{-\eta s} \sqrt{1+4^{-\left(\beta-\frac{\pi}{2}\right) s}} d s}{\cosh \eta} \cdot \frac{\cosh \eta}{\cosh \left(\eta+\frac{1}{2}\left(\beta-\frac{\pi}{2}\right)\right)} \\
& =: \tilde{F}(\eta) \cdot P(\eta) .
\end{aligned}
$$

The factor $P(\eta)$ in the above formulas is clearly bounded and it is easily verified that all its derivatives decay exponentially.

In order to show that $\tilde{Q}(\xi, \eta)$ is a Marcinkiewicz multiplier, we write

$$
\begin{aligned}
\tilde{Q}(\xi, \eta) & =1+\left(\beta-\frac{\pi}{2}\right)\left[\frac{2 \frac{\tanh \eta}{\eta}}{2 \pi \frac{\tanh \xi}{\xi}+\left(\beta-\frac{\pi}{2}\right) F(\eta)}-\frac{F(\eta)}{2 \pi \frac{\tanh \xi}{\xi}+\left(\beta-\frac{\pi}{2}\right) F(\eta)}\right] \\
& =: 1+\left(\beta-\frac{\pi}{2}\right)\left[\tilde{Q}_{1}(\xi, \eta)+\tilde{Q}_{2}(\xi, \eta)\right] .
\end{aligned}
$$

Therefore, we reduced our problem to prove that the functions $\tilde{Q}_{1}$ and $\tilde{Q}_{2}$ are Marcinkiewicz multipliers.

We now explicitly study the decay of the derivatives of the function $F(\eta)$. Once the behavior of $F(\eta)$ is known, it is straightforward to prove that $\tilde{Q}$, hence $Q$, is a multiplier.

It is immediately seen that there exist two positive constants $c_{1}, c_{2}$ such that

$$
c_{1} \frac{\tanh \eta}{\eta} \leq F(\eta) \leq c_{2} \frac{\tanh \eta}{\eta}
$$

Next, we focus on the derivative of $F(\eta)$ and we may just consider the factor $\tilde{F}(\eta)$ above.

We assume $\eta>0$, the case $\eta<0$ can be treated in the same fashion. We write

$$
\begin{aligned}
\tilde{F}(\eta) & =\frac{\int_{0}^{1} e^{-\eta s} \sqrt{1+4 e^{-\left(\beta-\frac{\pi}{2}\right) s}} d s+\int_{0}^{1} e^{\eta s} \sqrt{1+4 e^{\left(\beta-\frac{\pi}{2}\right) s}} d s}{\cosh \eta} \\
& =\frac{2}{1+e^{-2 \eta}}\left(\int_{0}^{1} e^{-\eta(s+1)} \sqrt{1+4^{-\left(\beta-\frac{\pi}{2}\right) s}} d s+\int_{0}^{1} e^{\eta(s-1)} \sqrt{1+4 e^{\left(\beta-\frac{\pi}{2}\right) s}} d s\right) \\
& =: I(\eta)+I I(\eta) .
\end{aligned}
$$

Now, $\frac{d I}{d \eta}(\eta)=A(\eta)-B(\eta)$, where

$$
\begin{aligned}
& A(\eta)=\frac{4 e^{-2 \eta}}{\left(1+e^{-2 \eta}\right)^{2}} \int_{0}^{1} e^{-\eta(s+1)} \sqrt{1+4 e^{\left(-\beta-\frac{\pi}{2}\right) s}} d s \\
& B(\eta)=\frac{2}{1+e^{-2 \eta}} \int_{0}^{1}(s+1) e^{-\eta(s+1)} \sqrt{1+4 e^{-\left(\beta-\frac{\pi}{2}\right) s}} d s .
\end{aligned}
$$


The term $A(\eta)$ decays exponentially, while

$$
\begin{aligned}
|B(\eta)| & \leq \frac{1}{1+e^{-2 \eta}} \int_{0}^{2} \tau e^{-\eta \tau} \sqrt{1+4 e^{-\left(\beta-\frac{\pi}{2}\right)(\tau-1)}} d \tau \\
& \leq \frac{C}{\eta^{2}} \int_{0}^{2 \eta} t e^{-t} d t \\
& \leq \frac{C}{\eta^{2}}
\end{aligned}
$$

Therefore, we conclude that $\left|\frac{d I}{d \eta}(\eta)\right| \leq \frac{C}{\eta^{2}}$. Similarly, we obtain the same estimate for $\frac{d I I}{d \eta}(\eta)$, so we conclude that $\left|\tilde{F}^{\prime}(\eta)\right| \leq \frac{C}{\eta^{2}}$.

Arguing as above, the estimate $\left|\tilde{F}^{\prime \prime}(\eta)\right| \leq \frac{C}{|\eta|^{3}}$ can be obtained, but we leave the details to the reader.

Finally, using the estimates we have on $\tilde{F}$ and its derivatives, it is now straightforward to prove that $\tilde{Q}$, thus $Q$, is a Marcinkiewicz multiplier and conclude the proof.

4.3. Proof of Lemma 3.3. We need to show that $m_{\alpha}$ given by (30) is a Marcinkiewicz multiplier in $\mathbb{R}^{2}$. Notice that since $\alpha>0$, in the variable $\eta$ we only need to estimate the decay for $\eta>0$. For, let $\varrho$ be a non-negative $C^{\infty}$ function on $\mathbb{R}, \varrho=1$ for $\eta \geq 1$ and $\varrho=0$ for $\eta \leq 0$. Then $(1-\varrho) m_{\alpha}$ is a Schwartz function in $\mathbb{R}^{2}$ uniformly in $\alpha$, and we only need to consider $\varrho(\eta) \cdot m_{\alpha}(\xi, \eta)$. However, $\varrho^{\prime}$ is compactly supported, so for simplicity of notation we just consider $m_{\alpha}$ and $\eta \geq 1$.

We now notice that for every non-negative integer $k$ there exists $C=C_{k}>0$ such that

$$
\left|m_{\alpha}(\xi, \eta)\right| \leq \frac{\eta e^{\alpha \eta}}{\sinh \eta}=\left|\frac{\eta^{k+1} e^{\alpha \eta}}{\sinh \eta}\right| \frac{1}{|\eta|^{k}} \leq \frac{C_{k}}{(1-\alpha)^{k+1}} \frac{1}{|\eta|^{k}} .
$$

Hence, in order to prove our lemma we need to estimate all the derivatives up to order two of $m_{\alpha}(\xi, \eta)$. Recall that $D(\xi, \eta)$ is defined in (28). We have

$$
\begin{aligned}
\partial_{\xi} m_{\alpha}(\xi, \eta) & =e^{\xi} e^{\alpha \eta} \frac{D(\xi, \eta)-\partial_{\xi} D(\xi, \eta)}{D^{2}(\xi, \eta)} \\
& =m_{\alpha}(\xi, \eta)\left[1-\frac{\partial_{\xi} D(\xi, \eta)}{D(\xi, \eta)}\right] \\
& =\frac{m_{\alpha}(\xi, \eta)}{D(\xi, \eta)}\left[\frac{\sinh \eta}{\eta} e^{-\xi}-\cosh \eta \frac{e^{-\xi}}{\xi}+\cosh \eta \frac{\sinh \xi}{\xi^{2}}\right] \\
& =\frac{e^{\alpha \eta}}{\left[\cosh \eta \frac{\sinh \xi}{\xi}+\frac{\sinh \eta}{\eta} \cosh \xi\right]^{2}}\left[\frac{\sinh \eta}{\eta}-\frac{\cosh \eta}{\xi}+\cosh \eta \frac{\sinh \xi}{\xi^{2}} e^{\xi}\right] \\
& =\psi_{\alpha}(\xi, \eta)\left[\frac{\sinh \eta}{\eta}-\frac{\cosh \eta}{\xi}+\cosh \eta \frac{\sinh \xi}{\xi^{2}} e^{\xi}\right]
\end{aligned}
$$

where

$$
\psi_{\alpha}(\xi, \eta)=\frac{e^{\alpha \eta}}{D^{2}(\xi, \eta)}
$$


Hence, recalling (28) and using the Cauchy-Schwarz inequality we have,

$$
\begin{aligned}
\left|\partial_{\xi} m_{\alpha}(\xi, \eta)\right| & \leq \frac{e^{\alpha \eta}}{D(\xi, \eta)} \cdot \frac{\left|\frac{\sinh \eta}{\eta}-\frac{\cosh \eta}{\xi}+\cosh \eta \frac{\sinh \xi}{\xi^{2}} e^{\xi}\right|}{D(\xi, \eta)} \\
& =\frac{\eta e^{\alpha \eta}}{\sinh \eta} \cdot \frac{1}{|\xi|} \cdot \frac{\frac{\sinh \eta}{\eta}\left|\xi \frac{\sinh \eta}{\eta}-\cosh \eta+\cosh \eta \frac{\sinh \xi}{\xi} e^{\xi}\right|}{D(\xi, \eta)^{2}} \\
& \leq \frac{C}{1-\alpha} \cdot \frac{1}{|\xi|} .
\end{aligned}
$$

For later reference, we observe that from (31) (with $k=1$ ) we also have

$$
\left|\partial_{\xi} m_{\alpha}(\xi, \eta)\right| \leq \frac{C}{(1-\alpha)^{2}} \frac{1}{|\xi \eta|} .
$$

Next,

$$
\begin{aligned}
& \partial_{\xi}^{2} m_{\alpha}(\xi, \eta) \\
& =\partial_{\xi} \psi_{\alpha}(\xi, \eta)\left[\frac{\sinh \eta}{\eta}-\frac{\cosh \eta}{\xi}+\cosh \eta \frac{\sinh \xi}{\xi^{2}} e^{\xi}\right] \\
& +\psi_{\alpha}(\xi, \eta)\left[\frac{\cosh \eta}{\xi^{2}}+\cosh \eta \frac{\cosh \xi}{\xi^{2}} e^{\xi}-2 \cosh \eta \frac{\sinh \xi}{\xi^{3}} e^{\xi}+\cosh \eta \frac{\sinh \xi}{\xi^{2}} e^{\xi}\right] \\
& =\psi_{\alpha}(\xi, \eta)\left\{\frac{-2\left[\cosh \eta\left(\frac{\cosh \xi}{\xi}-\frac{\sinh \xi}{\xi^{2}}\right)+\frac{\sinh \eta}{\eta} \sinh \xi\right]}{D(\xi, \eta)}\left[\frac{\sinh \eta}{\eta}-\frac{\cosh \eta}{\xi}+\cosh \eta \frac{\sinh \xi}{\xi^{2}} e^{\xi}\right]\right. \\
& \left.+\left[\frac{\cosh \eta}{\xi^{2}}+\cosh \eta \frac{\cosh \xi}{\xi^{2}} e^{\xi}-2 \cosh \eta \frac{\sinh \xi}{\xi^{3}} e^{\xi}+\cosh \eta \frac{\sinh \xi}{\xi^{2}} e^{\xi}\right]\right\} \\
& =\frac{\eta e^{\alpha \eta}}{\sinh \eta} \cdot \frac{1}{\xi^{2}} \cdot \frac{\frac{\sinh \eta}{\eta}}{D^{3}(\xi, \eta)}\left\{-2\left[\cosh \eta\left(\cosh \xi-\frac{\sinh \xi}{\xi}\right)+\frac{\sinh \eta}{\eta} \xi \sinh \xi\right]\right. \\
& \times\left[\xi \frac{\sinh \eta}{\eta}-\cosh \eta+\cosh \eta \frac{\sinh \xi}{\xi} e^{\xi}\right] \\
& +\left[\cosh \xi \frac{\sinh \eta}{\eta}+\cosh \eta \frac{\sinh \xi}{\xi}\right] \\
& \left.\times\left[\cosh \eta+\cosh \eta \cosh \xi e^{\xi}-2 \cosh \eta \frac{\sinh \xi}{\xi} e^{\xi}+\cosh \eta \sinh \xi e^{\xi}\right]\right\} \\
& =\frac{\eta e^{\alpha \eta}}{\sinh \eta} \cdot \frac{1}{\xi^{2}} \cdot \frac{2 \frac{\sinh \eta}{\eta}}{D^{3}(\xi, \eta)}\left\{\cosh \eta \frac{\sinh \eta}{\eta} \xi e^{-\xi}+\cosh ^{2} \eta \cosh \xi+\cosh \eta \frac{\sinh \eta}{\eta}\left(e^{\xi}+\sinh \xi\right)\right. \\
& \left.-\cosh \xi \cosh \eta \frac{\sinh \xi}{\xi} \frac{\sinh \eta}{\eta} e^{\xi}-\cosh ^{2} \eta \frac{\sinh \xi}{\xi}\right\} \text {. }
\end{aligned}
$$

Then, by (31) (with $k=0$ ) we have

$$
\left|\partial_{\xi}^{2} m_{\alpha}(\xi, \eta)\right| \leq \frac{C}{1-\alpha} \frac{1}{|\xi|^{2}} .
$$


Next,

$$
\begin{aligned}
\partial_{\eta} m_{\alpha}(\xi, \eta) & =\frac{m_{\alpha}(\xi, \eta)}{D(\xi, \eta)}\left[\frac{\sinh \xi}{\xi}(\alpha \cosh \eta-\sinh \eta)+\frac{\cosh \xi}{\eta}(\alpha \sinh \eta-\cosh \eta)+\cosh \xi \frac{\sinh \eta}{\eta^{2}}\right] \\
& =\frac{m_{\alpha}(\xi, \eta)}{D(\xi, \eta)}\left[(\alpha-1) D(\xi, \eta)+e^{-\eta}\left(\frac{\sinh \xi}{\xi}-\frac{\cosh \xi}{\eta}\right)+\cosh \xi \frac{\sinh \eta}{\eta^{2}}\right] \\
& =(\alpha-1) m_{\alpha}(\xi, \eta)+\frac{m_{\alpha}(\xi, \eta)}{D(\xi, \eta)}\left[e^{-\eta}\left(\frac{\sinh \xi}{\xi}-\frac{\cosh \xi}{\eta}\right)+\cosh \xi \frac{\sinh \eta}{\eta^{2}}\right] \\
& =:\left[(\alpha-1)+\frac{\phi(\xi, \eta)}{D(\xi, \eta)}\right] m_{\alpha}(\xi, \eta) .
\end{aligned}
$$

Using (31) (with $k=0,1$ ) and the fact that $\eta \geq 1$ so that $|\phi / D| \leq C|\eta|^{-1}$, we obtain

$$
\left|\partial_{\eta} m_{\alpha}(\xi, \eta)\right| \leq \frac{C}{1-\alpha} \frac{1}{|\eta|} .
$$

Furthermore,

$$
\begin{aligned}
\partial_{\eta}^{2} m_{\alpha}(\xi, \eta) & =\left[(\alpha-1)+\frac{\phi(\xi, \eta)}{D(\xi, \eta)}\right] \partial_{\eta} m_{\alpha}(\xi, \eta)+\frac{m_{\alpha}(\xi, \eta)}{D^{2}(\xi, \eta)}\left[\partial_{\eta} \phi(\xi, \eta) D(\xi, \eta)-\phi(\xi, \eta) \partial_{\eta} D(\xi, \eta)\right] \\
& =: I+I I .
\end{aligned}
$$

From (35) it follows that

$$
I=\left[(\alpha-1)+\frac{\phi(\xi, \eta)}{D(\xi, \eta)}\right]^{2} m_{\alpha}(\xi, \eta) .
$$

Thus, using (31) (with $k=2,0$ ), we see that $|I| \leq \frac{C}{1-\alpha}|\eta|^{-2}$. An elementary but long computation shows that

$$
\begin{aligned}
I I=\frac{m_{\alpha}(\xi, \eta)}{\eta^{2} D^{2}(\xi, \eta)}\left[2 \cosh \xi \frac{\sinh \xi}{\xi}+\cosh ^{2} \xi\right. & -\frac{\sinh ^{2} \xi}{\xi^{2}} \\
& \left.-\cosh ^{2} \xi \frac{\sinh ^{2} \eta}{\eta^{2}}-2 \cosh \xi \cosh \eta \frac{\sinh \xi}{\xi} \frac{\sinh \eta}{\eta}\right] .
\end{aligned}
$$

which easily implies that $|I I| \leq \frac{C}{1-\alpha}|\eta|^{-2}$. Therefore,

$$
\left|\partial_{\eta}^{2} m_{\alpha}(\xi, \eta)\right| \leq \frac{C}{1-\alpha} \frac{1}{|\eta|^{2}}
$$

for a constant $C$ independent of $\alpha$.

Finally, from (35), with an elementary computation we obtain that

$$
\begin{aligned}
\partial_{\xi} \partial_{\eta} m_{\alpha}(\xi, \eta)=\partial_{\xi} m_{\alpha}(\xi, \eta) & {\left[(\alpha-1)+\frac{\phi(\xi, \eta)}{D(\xi, \eta)}\right] } \\
& +\frac{m_{\alpha}(\xi, \eta)}{D^{2}(\xi, \eta)}\left[\partial_{\xi} \phi(\xi, \eta) D(\xi, \eta)-\phi(\xi, \eta) \partial_{\xi} D(\xi, \eta)\right] \\
=\partial_{\xi} m_{\alpha}(\xi, \eta)\left[(\alpha-1)+\frac{\phi(\xi, \eta)}{D(\xi, \eta)}\right] & \\
& +\frac{m_{\alpha}(\xi, \eta)}{D^{2}(\xi, \eta)}\left[\frac{1}{\xi \eta}\left(1-\cosh \xi \frac{\sinh \xi}{\xi}\right)\left(1-\cosh \eta \frac{\sinh \eta}{\eta}\right)\right] .
\end{aligned}
$$


Using (34) and (33) we now deduce that

$$
\left|\partial_{\xi} \partial_{\eta} m_{\alpha}(\xi, \eta)\right| \leq \frac{C}{1-\alpha} \frac{1}{|\xi \eta|},
$$

for a constant $C$ independent of $\alpha$, and the lemma is proved.

4.4. Proof of Lemma 3.4. If $m_{\alpha}(\xi, \eta)$ denotes the function (30), for all non-negative integer $k$ we clearly have

$$
\left|m_{\alpha, \gamma}(\xi, \eta)\right| \leq\left|m_{\alpha}(\xi, \eta)\right| \leq \frac{C_{k}}{(1-\alpha)^{k+1}} \frac{1}{|\eta|^{k}} .
$$

By symmetry we also have the estimate

$$
\left|m_{\alpha, \gamma}(\xi, \eta)\right| \leq \frac{C_{k}}{(1-\gamma)^{k+1}} \frac{1}{|\xi|^{k}}
$$

Therefore,

$$
\left|m_{\alpha, \gamma}(\xi, \eta)\right| \leq C \min \left(\frac{1}{1-\gamma}, \frac{1}{1-\alpha}\right) .
$$

Recalling that $D(\xi, \eta)$ is given by (28), arguing as in (35) we obtain

$$
\partial_{\eta} m_{\alpha, \gamma}(\xi, \eta)=\left[(\alpha-1)+\frac{\phi(\xi, \eta)}{D(\xi, \eta)}\right] m_{\alpha, \gamma}(\xi, \eta) .
$$

Since $\left|m_{\alpha, \gamma}(\xi, \eta)\right| \leq\left|m_{\alpha}(\xi, \eta)\right|$, the previous lemma guarantees that $\left|\partial_{\eta} m_{\alpha, \gamma}(\xi, \eta)\right| \leq \frac{C}{1-\alpha}|\eta|^{-1}$. Moreover,

$$
\begin{aligned}
\left|m_{\alpha, \gamma}(\xi, \eta)\right| & \leq \frac{e^{\gamma \xi} e^{\alpha \eta}}{\frac{\sinh \xi}{\xi} \cosh \eta} \leq \frac{C}{(1-\gamma)|\eta|}\left|\frac{\eta e^{\alpha \eta}}{\cosh \eta}\right| \\
& \leq \frac{C}{(1-\gamma)(1-\alpha)} \frac{1}{|\eta|} .
\end{aligned}
$$

Inserting this and (38) into (39) we obtain

$$
\begin{aligned}
\left|\partial_{\eta} m_{\alpha, \gamma}(\xi, \eta)\right| & \leq \frac{C}{(1-\gamma)} \frac{1}{|\eta|}+\frac{C}{(1-\gamma)}\left|\frac{\phi(\xi, \eta)}{D(\xi, \eta)}\right| \\
& \leq \frac{C}{(1-\gamma)} \frac{1}{|\eta|} .
\end{aligned}
$$

Therefore,

$$
\left|\partial_{\eta} m_{\alpha, \gamma}(\xi, \eta)\right| \leq C \min \left(\frac{1}{1-\gamma}, \frac{1}{1-\alpha}\right) \frac{1}{|\eta|} .
$$

For the second order derivative with respect to $\eta$, similarly to (36), we obtain

$$
\begin{aligned}
& \partial_{\eta}^{2} m_{\alpha, \gamma}(\xi, \eta) \\
& \quad=\left[(\alpha-1)+\frac{\phi(\xi, \eta)}{D(\xi, \eta)}\right]^{2} m_{\alpha, \gamma}(\xi, \eta)+\frac{m_{\alpha, \gamma}(\xi, \eta)}{D^{2}(\xi, \eta)}\left[\partial_{\eta} \phi(\xi, \eta) D(\xi, \eta)-\phi(\xi, \eta) \partial_{\eta} D(\xi, \eta)\right],
\end{aligned}
$$


where $\phi$ is defined in (35). Thus, using again the fact that $m_{\alpha, \gamma}$ is bounded from above by $m_{\alpha}$, using the previous lemma, we can easily conclude (see (37) ) that $\left|\partial_{\eta}^{2} m_{\alpha, \gamma}(\xi, \eta)\right| \leq \frac{C}{1-\alpha}|\eta|^{-2}$, but also $\left|\partial_{\eta}^{2} m_{\alpha, \gamma}(\xi, \eta)\right| \leq \frac{C}{1-\gamma}|\eta|^{-2} ;$ hence

$$
\left|\partial_{\eta}^{2} m_{\alpha, \gamma}(\xi, \eta)\right| \leq C \min \left(\frac{1}{1-\gamma}, \frac{1}{1-\alpha}\right) \frac{1}{|\eta|^{2}} .
$$

By symmetry we have the estimates

$$
\left|\partial_{\xi} m_{\alpha, \gamma}(\xi, \eta)\right| \leq \min \left(\frac{1}{1-\gamma}, \frac{1}{1-\alpha}\right) \frac{1}{|\xi|},
$$

and

$$
\left|\partial_{\xi}^{2} m_{\alpha, \gamma}(\xi, \eta)\right| \leq \min \left(\frac{1}{1-\gamma}, \frac{1}{1-\alpha}\right) \frac{1}{|\xi|^{2}}
$$

Finally, for the mixed derivative, we obtain

$$
\begin{aligned}
\partial_{\xi} \partial_{\eta} m_{\alpha, \gamma}(\xi, \eta)=\partial_{\xi} m_{\alpha, \gamma}(\xi, \eta) & {\left[(\alpha-1)+\frac{\phi(\xi, \eta)}{D(\xi, \eta)}\right] } \\
+ & \frac{m_{\alpha, \gamma}(\xi, \eta)}{D^{2}(\xi, \eta)}\left[\frac{1}{\xi \eta}\left(1-\cosh \xi \frac{\sinh \xi}{\xi}\right)\left(1-\cosh \eta \frac{\sinh \eta}{\eta}\right)\right] .
\end{aligned}
$$

The estimate

now follows easily.

$$
\left|\partial_{\xi} \partial_{\eta} m_{\alpha, \gamma}(\xi, \eta)\right| \leq C \min \left(\frac{1}{1-\gamma}, \frac{1}{1-\alpha}\right) \frac{1}{|\xi \eta|}
$$

Finally, we can conclude that $m_{\alpha, \gamma}$ is a Marcinkiewicz multiplier whose norm of the associated multiplier operator is bounded by a constant times $\min \left(\frac{1}{1-\gamma}, \frac{1}{1-\alpha}\right)$. We conclude the proof by noticing that $\min \left(\frac{1}{1-\gamma}, \frac{1}{1-\alpha}\right) \leq \frac{2}{(1-\alpha)+(1-\gamma)}$.

\section{REFERENCES}

[1] D. E. Barrett. Behavior of the Bergman projection on the Diederich-Fornæss worm. Acta Math., 168(1-2):110, 1992.

[2] D. E. Barrett, D. Ehsani, and M. M. Peloso. Regularity of projection operators attached to worm domains. Doc. Math, 20:1207-1225, 2015.

[3] D. E. Barrett and S. Şahutoğlu. Irregularity of the Bergman projection on worm domains in $\mathbb{C}^{n}$. Michigan Math. J., 61(1):187-198, 2012.

[4] M. Christ. Global $C^{\infty}$ irregularity of the $\bar{\partial}$-Neumann problem for worm domains. J. Amer. Math. Soc., 9(4):1171-1185, 1996.

[5] K. Diederich and J. E. Fornaess. Pseudoconvex domains: an example with nontrivial Nebenhülle. Math. Ann., 225(3):275-292, 1977.

[6] L. Grafakos. Classical Fourier analysis, volume 249 of Graduate Texts in Mathematics. Springer, New York, second edition, 2008.

[7] Z. Harper. Laplace transform representations and Paley-Wiener theorems for functions on vertical strips. Doc. Math., 15:235-254, 2010.

[8] P. S. Harrington, M. M. Peloso, and A. S. Raich. Regularity equivalence of the Szegö projection and the complex Green operator. Proc. Amer. Math. Soc., 143(1):353-367, 2015.

[9] C. O. Kiselman. A study of the Bergman projection in certain Hartogs domains. In Several complex variables and complex geometry, Part 3 (Santa Cruz, CA, 1989), volume 52 of Proc. Sympos. Pure Math., pages 219-231. Amer. Math. Soc., Providence, RI, 1991.

[10] K. D. Koenig. On maximal Sobolev and Hölder estimates for the tangential Cauchy-Riemann operator and boundary Laplacian. Amer. J. Math., 124(1):129-197, 2002. 
[11] S. G. Krantz and M. M. Peloso. Analysis and geometry on worm domains. J. Geom. Anal., 18(2):478-510, 2008.

[12] S. G. Krantz and M. M. Peloso. The Bergman kernel and projection on non-smooth worm domains. Houston J. Math., 34(3):873-950, 2008.

[13] S. G. Krantz, M. M. Peloso, and C. Stoppato. Completeness on the worm domain and the Müntz-Szász problem for the Bergman space. arXiv:1509.06383, 2015.

[14] S. G. Krantz, M. M. Peloso, and C. Stoppato. Bergman kernel and projection on the unbounded worm domain. Ann. Sc. Norm. Pisa, Oct. to appear 2016.

[15] L. Lanzani and E. M. Stein. Hardy spaces of holomorphic functions for domains in $\mathbb{C}^{n}$ with minimal smoothness. arXiv:1506.03748, June 2015.

[16] L. Lanzani and E. M. Stein. The Cauchy-Szegö projection for domains in $\mathbb{C}^{n}$ with minimal smoothness. arXiv:1506.03965, June 2015.

[17] J. D. McNeal and E. M. Stein. The Szegő projection on convex domains. Math. Z., 224(4):519-553, 1997.

[18] A. Monguzzi. A comparison between the Bergman and Szegö kernels of the non-smooth worm domain $D_{\beta}^{\prime}$. Complex Analysis and Operator Theory, pages 1-27, 2015.

[19] A. Monguzzi. Hardy spaces and the Szegö projection of the non-smooth worm domain $D_{\beta}^{\prime}$. J. Math. Anal. Appl., 436(1):439-466, 2016.

[20] A. Monguzzi and M. M. Peloso. Sharp estimates for the Szegö projection of Hardy spaces on the distinguished boundary of model worm domains. In preparation.

[21] A. Nagel, J.-P. Rosay, E. M. Stein, and S. Wainger. Estimates for the Bergman and Szegő kernels in $\mathbf{C}^{2}$. Ann. of Math. (2), 129(1):113-149, 1989.

[22] D. H. Phong and E. M. Stein. Estimates for the Bergman and Szegö projections on strongly pseudo-convex domains. Duke Math. J., 44(3):695-704, 1977.

Dipartimento di Matematica "F. Enriques", Università degli Studi di Milano, Via C. Saldini 50, I-20133 Milano

E-mail address: alessandro.monguzzi@unimi.it

E-mail address: marco.peloso@unimi.it 